\title{
Title: Systematic measurement of combination drug landscapes to predict in vivo treatment outcomes for tuberculosis
}

\author{
Authors: Jonah Larkins-Ford ${ }^{1-4}$, Talia Greenstein ${ }^{1-3}$, Nhi Van ${ }^{1}$, Yonatan N. Degefu ${ }^{1,4}$, Michaela \\ C. Olson ${ }^{1}$, Artem Sokolov ${ }^{4}$, Bree B. Aldridge ${ }^{1-5 *}$
}

\begin{abstract}
Affiliations:
${ }^{1}$ Department of Molecular Biology and Microbiology, Tufts University School of Medicine, Boston, MA.

${ }^{2}$ Stuart B. Levy Center for Integrated Management of Antimicrobial Resistance, Boston, MA.

${ }^{3}$ Graduate School of Biomedical Sciences, Tufts University School of Medicine, Boston, MA.

${ }^{4}$ Laboratory of Systems Pharmacology, Harvard Medical School, Boston, MA.

${ }^{5}$ Department of Biomedical Engineering, Tufts University School of Engineering, Medford, MA.

*To whom correspondence should be addressed: Bree B. Aldridge, bree.aldridge@tufts.edu, 617-636-6703
\end{abstract}

One Sentence Summary: Signatures of in vitro potency and drug interaction measurements predict combination therapy outcomes in mouse models of tuberculosis. 
Abstract: A lengthy multidrug chemotherapy is required to achieve a durable cure in tuberculosis. Variation in Mycobacterium tuberculosis drug response is created by the differing microenvironments in lesions, which generate different bacterial drug susceptibilities. To better realize the potential of combination therapy to shorten treatment duration, multidrug therapy design should deliberately explore the vast combination space. We face a significant scaling challenge in making systematic drug combination measurements because it is not practical to use animal models for comprehensive drug combination studies, nor are there well-validated highthroughput in vitro models that predict animal outcomes. We hypothesized that we could both prioritize combination therapies and quantify the predictive power of various in vitro models for drug development using a dataset of drug combination dose responses measured in multiple in vitro models. We systematically measured M. tuberculosis response to all 2- and 3-drug combinations among ten antibiotics in eight conditions that reproduce lesion microenvironments. Applying machine learning to this comprehensive dataset, we developed classifiers predictive of multidrug treatment outcome in a mouse model of disease relapse. We trained classifiers on multiple mouse models and identified ensembles of in vitro models that best describe in vivo treatment outcomes. Furthermore, we found that combination synergies are less important for predicting outcome than metrics of potency. Here, we map a path forward to rationally prioritize combinations for animal and clinical studies using systematic drug combination measurements with validated in vitro models. Our pipeline is generalizable to other difficult-to-treat diseases requiring combination therapies.

\section{[Main Text: ]}

\section{Introduction}


Tuberculosis (TB), caused by infection with Mycobacterium tuberculosis (Mtb), remains a major global health issue. In 2019, an estimated ten million people fell ill with TB, and about 1.4 million people died (1). Development of shorter treatment regimens is a key part of the third pillar of the WHO End TB Strategy (2). Multidrug treatment regimens were developed to treat active TB infections by shortening treatment duration, reducing disease relapse, and decreasing antibiotic resistance development (3). The standard TB treatment is six to nine months of multidrug treatment with an estimated $85 \%$ cure rate $(1,4,5)$. The first two months of treatment (intensive, bactericidal phase) consist of four drugs (isoniazid, rifampicin, pyrazinamide, and ethambutol) that reduce sputum Mtb levels but are less effective against non-replicative bacilli $(3,4,6)$. The following four to seven months of treatment (continuation phase) consist of two drugs (isoniazid and rifampicin) aimed at reducing disease relapse by treating persisting bacteria that survived the intensive phase $(3,4,6)$. New regimens that can more efficiently treat Mtb are needed to shorten the intensive phase of treatment and reduce or eliminate the bacteria that persist and require continuation phase treatment (4).

Due, in large part, to the heterogeneity of TB lesions and treatment response among the Mtb population, combination therapy is required to treat active TB. Therapies should therefore be designed as combinations of antibiotics rather than single antibiotics alone. There are many drug options for new treatment regimens using existing drugs and drugs in development (7), which creates an enormous number of possible drug combinations (5). Preliminary results from a Phase 3 clinical trial ("Study 31") demonstrated that treatment could be shortened using a novel combination of existing TB antibiotics $(8,9)$. Relatively new drugs that can target non-replicative bacteria (bedaquiline, pretomanid, delamanid, SQ109) (10-12) in combination with established drugs are in new, treatment-shorting regimens for multidrug resistant TB (MDR-TB) $(5,13,14)$. 
Treatment shortening potential in Phase $2 \mathrm{~b}$ trials $(15,16))$ led to the Phase 3 STAND clinical trial testing the use of pretomanid with pyrazinamide and moxifloxacin, PaMZ (Table 1) (17). Adding bedaquiline to PaMZ (BPaMZ, Table 1) in a Phase 2b trial (18) shortened culture conversion time of MDR-TB so dramatically that the STAND trial was put on permanent hold to start the Phase 3 SimpliciTB trial to evaluate BPaMZ for treating both drug-sensitive TB and $\operatorname{MDR}-\mathrm{TB}(9,19)$. Together, these studies and the history of TB drug regimen design has demonstrated that there is treatment-shortening potential in the drug combination space. A critical step for developing new treatment regimens is prioritizing the thousands of drug combinations before clinical testing. However, it is not practical to evaluate thousands of combinations using the current preclinical regimen design pipeline, which combines in vitro and small animal studies. An efficient methodology is needed to systematically assess drug combinations and prioritize the thousands of multidrug combinations for their treatment-shorting potential.

Animal models are critical to regimen development, and mouse models are a primary tool in multidrug therapy design (20-24). Mouse strains where Mtb is primarily intracellular (e.g., BALB/c and C57BL/6) are the most widely used (24). Mouse strains that form mixed lesion types (e.g., $\mathrm{C} 3 \mathrm{HeB} / \mathrm{FeJ}$ ) are used to study drug response because the disease pathology is more humanlike, include granulomas with caseous necrotic cores $(21,25,26)$. Mtb drug response differs between these two types of mouse models, and both are important preclinical tools because the model-specific drug response is thought to result from the different lesion microenvironments present in each animal model $(24,27-29)$. Despite their utility for regimen development, comprehensive drug measurements in mice are not feasible. It is only practical to perform systematic drug combination studies in vitro, but in vitro studies do not clearly map to in vivo outcomes $(24,30)$. Many in vitro models mimic aspects of the host microenvironment encountered 
in the different TB lesion types. Many of these in vitro models are well suited for systematic drug combination studies, but none have been validated to prioritize drug combinations against preclinical animal models.

We propose to realize the potential of drug combinations to improve treatment by developing a pipeline to map in vitro measurement of drug response to outcomes in mouse models. Here, we utilized the efficiency of an experimental design and analysis method called DiaMOND (diagonal measurement of n-way drug interactions) (31) to create a compendium of drug combination responses in Mtb using multiple in vitro models that were designed to reproduce aspects of the environments encountered in different lesion types. Applying machine learning to this comprehensive in vitro dataset, we identified signatures of drug potency and interaction that could predict whether combinations would outperform the standard of care. Classifiers based on these signatures also enabled us to establish a mapping between in vitro models and the different mouse models, which differ in lesion type (microenvironment) and outcome. Overall, our study establishes a logistical path to optimize combination therapies for TB using systematic measurement in validated in vitro growth models and computational modeling.

\section{Results}

\section{Drug combination compendium construction}

We developed a pipeline to efficiently prioritize drug combinations early in regimen development based on drug combination measurements from in vitro models. Using the DiaMOND methodology (31), we designed a compendium of drug combination measurements to survey informative drug-dose combinations (DiaMOND compendium). To compare in vitro data to 
treatment responses in animal models, our DiaMOND compendium focused on (A) first- and second-line agents, for which there are abundant animal data and (B) measurements in in vitro growth conditions that model environments encountered during infection.

Mtb encounters a diversity of environmental niches during infection that influence response to drug treatment. We aimed to model drug response by aggregating measurements from a suite of in vitro models. We focused on modeling factors previously shown to influence Mtb growth and/or drug response, such as different carbon sources and abundance, low $\mathrm{pH}$, low oxygen tension, and the intracellular environment $(21,30,32-40)$. We developed or adapted eight in vitro models that were reproducible and scalable for systematic, high-throughput drug combination studies for this study. We varied carbon sources, with an emphasis on cholesterol and fatty acids, to model the lipid-rich environment in TB granulomas, using butyrate, valerate, cholesterol, and higher levels of cholesterol (cholesterol-high) as sole carbon sources. We used 7H9-based medium to compare against the most commonly utilized in vitro growth model with glycerol as a carbon source (standard). We also included in vitro models that mimic important factors encountered during infection: low pH (acidic), infection of J774 macrophages (intracellular), and developed a lowoxygen multi-stress model that induces dormancy using butyrate as a carbon source, sodium nitrate to respire (41-43), and plate seals to limit oxygen (dormancy). The doubling times varied considerably among the models, ranging from $16 \mathrm{~h}$ to one week (Fig. 1A). We scaled the timing of the experiments relative to the doubling time of each model so that drug response measurements would not be biased by changes in growth rate (Table S1).

\section{Drug combination dose response measurements}

For the DiaMOND compendium, we selected ten antibiotics in first- and second-line TB treatment regimens and for which there are abundant in vivo (mouse) data (Table 1, Table S2). These drugs 
include cell wall synthesis inhibitors (ethambutol, isoniazid, and pretomanid), rifamycin transcriptional inhibitors (rifampicin and rifapentine), protein synthesis inhibitor (linezolid), inhibitors of energy metabolism (bedaquiline and clofazimine), DNA replication inhibitor (moxifloxacin), and the antimycobacterial agent pyrazinamide (Fig. 1A, Table S2). We treated the Mtb Erdman strain carrying an autoluminescent reporter and measured both optical density $\left(\mathrm{OD}_{600}\right)$ and luminescence at multiple time points after drug treatment. We observed a strong dependency in drug potency on in vitro model (Fig. 1A, inhibitory concentration to achieve 90\% inhibition, $\mathrm{IC}_{90}$. Table S3), consistent with the idea that drug efficacy is influenced by bacterial stress (44). We did not observe remarkable correlations in potency profiles by in vitro model. However, hierarchical clustering of drug potencies showed some groupings of drugs consistent with their target cell process (e.g., rifamycin transcriptional inhibitors group together, isoniazid and pretomanid - inhibitors of cell wall synthesis - group together). We also observed clustering of similar in vitro models. For example, potency profiles from growth media with short-chain fatty acids butyrate and valerate as the carbon source group together (Fig. 1A).

We observed condition-specific drug potencies consistent with previous reports, suggesting that the models we adapted for high-throughput drug response measurements may be predictive of outcomes in animals. For example, the activity of pyrazinamide in acidic and intracellular models and inactivity in the standard model (Table S2) was consistent with in vitro $(45,46)$ and animal studies (47-49). We also observed pyrazinamide activity with lipid carbon sources, which has not been previously reported. As previously described, the rifamycins shared similar potency profiles with higher potency of rifapentine (Table S3) (50). Bedaquiline was more potent in medium with lipids as the carbon source compared to standard medium with sugars as previously described (51). Isoniazid potency was lower in the dormancy model, consistent with its inactivity towards non- 
replicating bacilli (52-54) and previous studies showing decreased efficacy in the presence of nitrite (41). The wide range of single-drug responses and consistency with prior studies suggest that the in vitro models in this study produce non-redundant drug response data and form a validated set of conditions to model the lesion-specific variation in drug response.

Using these eight in vitro models, we constructed a compendium of systematic drug combination measurements by utilizing the DiaMOND method's efficiency (Box). DiaMOND is a geometric optimization of the traditional checkerboard assay of drug-dose combinations. DiaMOND estimates the effect of combining drugs using a fraction of possible drug-dose combinations and focuses on the single drug and equipotent drug combination dose responses (31). We measured all 1-, 2-, and 3-drug combination dose responses (totaling 175 combinations) in at least biological duplicate (Fig. 1B), resulting in a compendium of over 51,000 individual dose response curves. We focused our analysis on up to two timepoints per in vitro model to navigate this complex dataset. We chose the last time point (terminal, T) that is relative to the doubling rate (4 to 5 doublings for most models) and at a consistent treatment timepoint (constant, C) across in vitro models, $\sim 7$ days post treatment; Fig. 1A, Table S1. We also selected the measurement type that best benchmarks against colony forming units $\left(\mathrm{OD}_{600}\right.$ for all models except intracellular and dormancy models, for which we used luminescence, Fig. S1). This selected dataset represents approximately one-quarter of the total number of compendium dose responses.

We analyzed the single- and combination-drug treatments to derive potency and drug interaction information (see Box). With DiaMOND, we can quantify the degree and directionality of interactions at different growth inhibition levels using common null models (e.g., Loewe additivity and Bliss independence). Drug combinations that are more or less effective than expected based on single-drug behaviors are considered synergistic and antagonistic, respectively. Drug 
interactions are quantified with fractional inhibitory concentrations (FICs) at different growth inhibition levels (e.g., $\mathrm{FIC}_{50}$ and $\mathrm{FIC}_{90}$ are measured at the $\mathrm{IC}_{50}$ and $\mathrm{IC}_{90}$, respectively). FIC measurements were log-transformed to represent synergistic and antagonistic combinations with negative and positive $\log _{2}$ (FIC) values, respectively. Drug interaction metrics based on Loewe additivity and Bliss independence were correlated ( $\mathrm{FIC}_{50}$ and $\mathrm{FIC}_{90}$ for the constant and terminal time points, $r=0.81, \mathrm{p}<2.2 \times 10^{-308}$, Pearson's correlation, Fig. S2); we, therefore, selected Loewe additivity as the null model for systematic analysis in the compendium ( $\mathrm{FIC}_{50}$ and $\mathrm{FIC}_{90}$ ). Dose response curves provide treatment potency metrics at a low dose $\left(\mathrm{AUC}_{25}\right.$; a normalized area under the curve to $\mathrm{IC}_{25}$, see Box) or high dose ( $\mathrm{E}_{\mathrm{inf}}$; the maximum achievable effect). To compare potency across models where Mtb have different growth properties, we calculated the maximum achievable inhibition of normalized growth rate (GR $\mathrm{Gnf}_{\text {; }}$ see Box), which allows direct comparison of treatment effects on cells with very different growth rates (55). Though many other drug response metrics may be calculated from DiaMOND data, our analysis focused on these five metrics -- FIC 50 , FIC90, $\mathrm{AUC}_{25}, \mathrm{E}_{\mathrm{inf}}, \mathrm{GR}_{\text {inf }}$-- because they represent well-characterized and biologically interpretable aspects of drug interactions and potencies across low- and high-dose ranges.

\section{Drug synergy is uncommon and does not distinguish effective combinations}

To identify patterns in drug interactions, we clustered the compendium drug interactions at the terminal time point in all eight growth environments, using $90 \%$ growth inhibition $\left(\log _{2}\left(\mathrm{FIC}_{90}\right)\right.$, Fig. 2A) and $50 \%$ growth inhibition $\left(\log _{2}\left(\mathrm{FIC}_{50}\right)\right.$, Fig. S3). Clustering did not reveal obvious model-wide synergy for any combination. Instead, we observed that most drug interactions were antagonistic $\left(70 \%\right.$ of $\left.\mathrm{FIC}_{90}>0\right)$, consistent with a general trend towards antagonism in drug interactions observed in other organisms $(31,56-60)$ and cancer (61). The tendency towards antagonism depended on the growth model, with some conditions showing a balance between 
synergy and antagonism (intracellular and acidic), and others almost entirely antagonistic (cholesterol). Together these data suggest that synergy is a property of both drug and growth environment rather than an intrinsic property of the drug.

To understand whether combinations that tend toward in vitro synergy are more effective in vivo, we compared selected combinations with differences in disease relapse from the most commonly used mouse strains (e.g., BALB/c, C56BL/6, Swiss). The relapsing mouse models (RMM) evaluate drug efficacy months after cessation of drug treatment, somewhat analogous to the clinical measurement of relapse $(62,63)$. We did not observe combination rank-ordering by synergy in any growth condition that matched efficacy in the RMM; e.g., BPaL $>$ MRZ $>H R Z>R Z$ (Fig. 2B) (22, 64-71). Instead, we observed the 3-drug standard of care (HRZ) was the most synergistic drug combination, and $\mathrm{BPaL}$ was the most antagonistic among this subset (Fig. 2B). These examples suggest that drug interaction scores alone in the measured in vitro models were poor indicators of in vivo combination efficacy.

Synergistic drug combinations are not necessarily more effective than antagonistic combinations as the maximum effect of a combination can change independently of the drug interaction (72) (see Box). A tradeoff between synergy and efficacy appears to be important to consider when selecting effective drug combinations for treating other diseases (e.g., hepatitis C, HIV, and cancer), with maximum effect often being more important than synergy $(73,74)$. To determine if the maximum effect could be used to prioritize combinations from the DiaMOND compendium, we clustered the $\mathrm{E}_{\text {inf }}$ (a measure of maximum dose response effect, see Box) for all compendium drug combinations in all eight in vitro models at the terminal time point (Fig. 2C). We observed a high maximum effect ( $E_{\text {inf }}>0.9$, Fig. $2 \mathrm{C}$ ) in most combinations, consistent with the drugs' known anti-Mtb effects. Dormancy and cholesterol-high models exhibited little variation in $E_{\text {inf, }}$ 
suggesting that neither condition had the dynamic range of maximum effect needed to discriminate among combinations or that all drug combinations are effective in these growth conditions for extended drug exposures. We compared $\mathrm{E}_{\text {inf }}$ profiles for the selected combinations we examined before, and we found that BPaL was more potent than HRZ or MRZ (Fig. 2D), consistent with animal outcomes of these regimens $(22,64-71)$. These examples suggest that maximum achievable effect in vitro may be a stronger predictor of outcomes in mouse models than synergy. As with

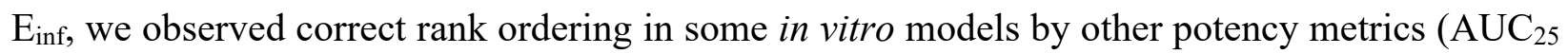

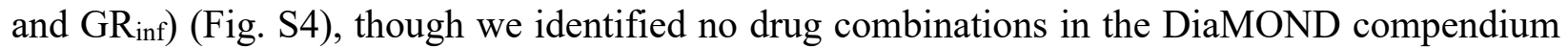
that were maximally potent across all eight models (Fig. S5). The correct ordering of selected drug combinations by mouse outcome suggests that the DiaMOND compendium contains useful information for identifying efficacious drug combinations.

DiaMOND metric signatures are predictive of treatment outcomes in the relapsing mouse model

We hypothesized that combinations of in vitro measurements could be compiled to model the in vivo microenvironments experienced by Mtb during drug treatment. We asked whether signatures of DiaMOND compendium measurements could distinguish drug combinations that were better than the standard of care in animal studies, HRZE or HRZ (Table 1). We classified 27 drug combinations that we measured in the compendium based on whether the treatment outcome in published RMM studies was better than the standard of care (C1) or not (C0) (Table S4). Principal component analysis (PCA) demonstrated that linear combinations of in vitro features could separate $\mathrm{C} 0$ and $\mathrm{C} 1$ drug combinations (Fig. 3A, Wilcoxon rank-sum, $\mathrm{p}<0.005$, Table S5). Inspection of feature contributions to the principal component $(\mathrm{PC})$ that best separates $\mathrm{C} 0$ and $\mathrm{C} 1$ drug combinations revealed many features related to cholesterol, standard, and valerate growth models (Fig. 3B). We also observed that potency metrics $\left(\mathrm{AUC}_{25}, \mathrm{E}_{\mathrm{inf}}, \mathrm{GR}_{\text {inf }}\right)$ are almost 
exclusively represented in the top 20 contributing features (Fig. 3B). Together these results suggest that effective separation of $\mathrm{C} 1$ and $\mathrm{C} 0$ drug combinations requires measurement of drug combination potency in multiple growth environments.

To develop signatures of DiaMOND metrics that characterize $\mathrm{C} 0$ from $\mathrm{C} 1$ combinations, we trained binary classifiers with eight different machine learning (ML) methods to distinguish $\mathrm{C} 0$ and $\mathrm{C} 1$ drug combinations and compared their performance in 5-fold cross-validation (Table S6). We observed that nonlinear ensemble methods (Bayesian additive regression trees, random forest (RF), and gradient boosted trees) outperformed other ML algorithms, as measured by the area under the receiver operator characteristic (ROC) curve (AUC) and the F1 statistic, which is the harmonic mean of precision and recall (Table S6). We performed additional validation of the RF model by applying it to higher-order (4- and 5-way) drug combinations commonly used in preclinical and clinical tests that were not considered during model training (Table S7). The RF model accurately predicted outcomes (Fig. $3 \mathrm{C}, \mathrm{AUC}=1, \mathrm{~F} 1=0.86$ ) and exhibited performance similar to what was estimated in cross-validation. Overall, the performance of ML models demonstrates that there is a strong signal in the DiaMOND compendium that is predictive of RMM drug combination efficacy.

We observed that some of the in vitro models in the DiaMOND compendium are well-represented among the top-ranked features in the classifying PCs (Fig. 3B). In contrast, other in vitro models are not present, suggesting that a subset of in vitro models may be sufficient to predict treatment outcome in the RMM. We asked whether classifiers using the DiaMOND compendium data from one in vitro model at a time were predictive of RMM outcome class. We observed that the data signal separating $\mathrm{C} 0$ and $\mathrm{C} 1$ drug combinations appeared in at least one $\mathrm{PC}$ for all eight in vitro models (Fig. 4A, Wilcoxon rank-sum test, $\mathrm{p}<0.05$, Fig. S6). Furthermore, the five technically 
simpler models to work with exhibited clear C0 and $\mathrm{C} 1$ separation (Fig. 4A, in vitro models cholesterol, butyrate, standard, valerate, Fig. S6 in vitro model acidic).

Though the single in vitro model classifiers were moderately predictive, they did not perform as well as the classifier trained using data from all eight in vitro models (Table S8). We asked whether another high-performing classifier could be derived using a subset of in vitro models. We systematically trained RF classifiers by considering all possible model combinations and observed that among the 255 possible combinations of in vitro models, 67 (26.3\%) performed better than the classifier trained on all eight models. Furthermore, predictors including only the simpler in vitro models performed as well or better than those including the "complex" (intracellular, dormancy, cholesterol high) models (Fig. 4B, student's t-test, $\mathrm{p}>0.05$ ). We further validated the highest performing classifiers trained on the simple in vitro models by applying them to the higherorder (4- and 5-way) drug combinations as well as drug combinations involving antibiotics (delamanid, sutezolid, and SQ109, Table S1) that were not included in the compendium's 10-drug set (Table S7). The high performance of classifiers on this validation set suggests that computationally combining simple in vitro models can produce classifiers that inform possible RMM outcomes (Fig. 4C). Additionally, the large number of classifiers that exhibit high accuracy and the shared DiaMOND compendium metrics among several in vitro models suggests that there may be multiple combinations of in vitro models that are predictive of outcomes in the RMM.

With many high performing RMM classifiers trained using subsets of the five simple in vitro models (Fig. 4C), we assessed whether the predicted RMM outcome for specific drug combinations would be consistent between these classifiers. The classifiers produce a probability that a drug combination belongs to each class (e.g., drug combination $\mathrm{X}$ belongs to $\mathrm{C} 1$ with $60 \%$ probability and $\mathrm{C} 0$ with $40 \%$ probability). The threshold probability is usually at $50 \%$ to assign 
the classification, but the probability can also rank combination classification likelihood. We tabulated the predicted probabilities of outcome for all combinations in the compendium, as well as the higher-order and new drug combination validation set, using the top-performing simple in vitro model classifiers shown in Fig. 4C. As we had previously observed, rank-ordering the percent probabilities within each classifier shows high predictive performance when evaluating the validation set. Among all predictions made for the compendium and validation combinations, we noted that $36 \%$ of drug combinations had discordant predictions among the three classifiers. We did not observe a consistent pattern in which a classifier was discordant. We next tested whether a consensus prediction could be generated by simply averaging the probabilities of the top three classifiers. We observed that the discordant combinations were clustered in the second quartile (probability of $\mathrm{C} 1$ around $25-50 \%$ ), suggesting that classifiers are most prone to error for combinations that are $\mathrm{C} 0$. This may be due to the mild class imbalance in the training set $(11 \mathrm{C} 0$ and $16 \mathrm{C} 1$ combinations). The consensus prediction was highly accurate ( $84 \%$ of validation set and $93 \%$ overall). Incorrect consensus predictions were at the border between $\mathrm{C} 0$ and $\mathrm{C} 1$ at 42 $47 \% \mathrm{C} 1$, indicating that the misclassification was due to ambiguity near the $50 \%$ decision boundary instead of strong classifier discordance. We conclude that a simple averaging of the probabilities generated by top classifiers is a practical means to construct an accurate consensus rank ordering for predicting drug combination response outcomes.

DiaMOND metrics describe the efficacy of drug combination treatments in the C3HeB/FeJ mouse model

Given the success of ML classifiers to predict RMM outcomes, we next asked whether the DiaMOND compendium can be used to predict outcomes in other mouse models. Bactericidal activity in the most commonly used mouse strains (e.g., BALB/c, C56BL/6, Swiss) has been used 
extensively to evaluate drug combination effectiveness. Bactericidal activity in these models (bactericidal mouse model, BMM, Table 1) measures the reduction in bacterial burden by drug treatment immediately following drug treatment and can be assessed more quickly than relapse. Using the same analysis pipeline, we trained $\mathrm{ML}$ classifiers to recognize $\mathrm{C} 0$ or $\mathrm{C} 1 \mathrm{drug}$ combinations for the BMM outcome (Table S9) but observed that the classifier performance was only mildly predictive $(\mathrm{AUC}=0.67, \mathrm{~F} 1=0.40)(\mathrm{Fig} . \mathrm{S} 7)$. Additional analysis of in vitro model subsets identified many predictors with improved performance, but this improvement did not generalize to test data. Moderate model training performance and poor generalizability to new data suggest that the drug combination information needed for BMM outcome predictions may be difficult to capture with the in vitro models developed and used in this study.

The $\mathrm{C} 3 \mathrm{HeB} / \mathrm{FeJ}(\mathrm{HeB})$ mouse strain has become important for $\mathrm{TB}$ regimen development because the disease pathology is more similar to humans than other mouse strains $(26,27,75)$. This includes the formation of caseous, necrotic granulomas that are characterized by low oxygen content (hypoxia) $(27,75,76)$ and differential drug penetrance $(77,78)$. These lesions also contain large numbers of extracellular, non-replicating bacteria $(24,76)$. Like other mouse studies, those with $\mathrm{HeB}$ mice use bactericidal $(\mathrm{BHeB})$ and relapse outcomes to determine drug effectiveness. Fewer drug combinations have been tested and published using HeB mice than other mouse strains. The DiaMOND compendium contained too few measured combinations to train ML classifiers. When we integrated the compendium combinations with higher-order drug combinations, we obtained a total of 16 combinations (Table S10) for the BHeB outcome, which was sufficient to train ML classifiers. However, we were not able to do the same for the relapse outcome, where we had four total combinations, even after augmenting with higher-order information. 
To understand if DiaMOND metrics distinguish $\mathrm{C} 0$ and $\mathrm{C} 1 \mathrm{BHeB}$ combinations using this expanded training dataset, we evaluated class separation with PCA. We observed significant separation of BHeB outcome classes along the third PC (PC3) (Fig. 5A, p<0.005, Table S11). We then examined the top 10 features in PC3 by contribution (Fig. 5B) and found that the in vitro models and metrics were distinct from those we observed in the RMM analysis (Fig. 3B). Notably, the metrics for the $\mathrm{BHeB}$ were entirely drug interactions (Fig. 5B), and the presence of the dormancy model in the top ten features was of particular interest because we expected hypoxiainduced dormancy to be a microenvironment specific to the $\mathrm{C} 3 \mathrm{HeB} / \mathrm{FeJ}$ mice $(27,75,76)$. Using the same approach described for RMM, we developed accurate RF models to classify BHeB C1 and $\mathrm{C} 0$ combinations (Fig $5 \mathrm{C}$, all in vitro models, $\mathrm{AUC}=0.9, \mathrm{~F} 1=0.80$ ). Systematic evaluation of RF classifiers using all possible combinations of in vitro model subsets revealed that complex models did not improve performance (Fig. S8). Specifically, we found that models without dormancy perform as well as those with it (Fig. S9). As with the RMM classifiers, we identified in vitro model subsets that performed better than all models together trained for the $\mathrm{BHeB}$ outcome (37 $(12.9 \%))$. Lipid and acidic in vitro models featured prominently among the most accurate classifiers. Together, these analyses demonstrate that the DiaMOND compendium data predicts outcomes in two pathologically distinct mouse models, suggesting that enough key information can be captured by simple in vitro models to prioritize combination therapies for animal model tests.

\section{Potency and antagonism are correlated with improved outcomes in mouse models}

The signatures of DiaMOND data describing outcomes in RMM and BHeB highlighted that potency metrics were key predictors for RMM, while drug interactions were key for BHeB outcome classification. To understand whether $\mathrm{C} 0$ and $\mathrm{C} 1$ drug combinations showed significant 
differences in these metrics, we examined the top five features from the most discriminatory PCs for both mouse models. Univariate analysis revealed significant differences between three of the top five features for the RMM outcome (Fig. 6A. Wilcoxon rank-sum, $p<0.05$ ). In each of these five features, potency was higher in $\mathrm{C} 1$ combinations than $\mathrm{C} 0$ combinations, which is consistent with expectations of increased potency for the most effective drug combinations. The top features describing $\mathrm{BHeB}$ outcomes are drug interactions (Fig. 6B), and three of the top five exhibited significant differences between $\mathrm{C} 0$ and $\mathrm{C} 1$ combinations (Wilcoxon rank-sum, $\mathrm{p}<0.05$ ). For all five drug interaction features, $\mathrm{C} 1$ was the more antagonistic class. That antagonistic drug combinations may be more favorable is consistent with the results of our comparison of BPaL to the standard of care (HRZ, Fig. 2B). We found that different metric types (potency or interactions) may provide information that maps to different outcome types (bactericidal or relapse) in animal studies. Furthermore, our analysis suggests that high potency and antagonism in in vitro assays may be characteristics of favorable drug combinations.

\section{Discussion}

Our goal in this study was to develop a pipeline to efficiently prioritize drug combinations early in the TB regimen design process. Most in vitro drug efficacy studies utilize single growth conditions, which have not been clearly mapped to in vivo outcomes $(24,30)$. Furthermore, conflicting results from multiple in vitro models have not been readily resolvable. We hypothesized that treatment efficacy in vivo could be modeled as a "sum-of-parts" of the complex microenvironment. Therefore, we generated a dataset that profiles drug combination effects against $\mathrm{Mtb}$ in eight different in vitro growth environments. With this comprehensive drug combination data compendium, we identified signatures of potencies and drug interactions in 
specific in vitro models that distinguish whether drug combinations are better than the standard of care in two important preclinical mouse models. We found that ML classifiers were accurate predictors of mouse disease relapse using data from only a few simple in vitro models. These classifiers were validated with higher-order (4- and 5-drugs) combinations and had predictive power for combinations with drugs not included in the model training. Together, our study establishes a practical approach to prioritize combination therapies using economical, scalable, and expandable in vitro measurements.

Synergy is often assumed to be a property of optimized combination therapies because synergistic drugs are more effective together than expected based on single-drug efficacies alone. Our mapping of the DiaMOND compendium onto outcomes in two different mouse models challenges this notion. In the relapsing mouse model, drug interactions were not key features for classification; instead, the potency measures from the drug dose response curves were the most important predictors of outcome (Fig. 3B). Our findings are consistent with reports of treatment in hepatitis $\mathrm{C}$, cancer, and $\operatorname{HIV}(73,74,79)$ that show a tradeoff between maximizing synergy and potency of a drug combination. Maximizing potency was often more important than synergy in treating these diseases with multidrug therapies $(73,74,79)$. Antagonism was prevalent in our compendium (Fig. 2A), and we found that antagonism was characteristic of more efficacious drug combinations for the $\mathrm{C} 3 \mathrm{HeB} / \mathrm{FeJ}$ bactericidal model (Fig. 6B, $\mathrm{C} 1$ more antagonistic than $\mathrm{C} 0$ ). Partnering the most potent drugs together during regimen design may be generating highly potent combinations but biasing these combinations towards antagonistic drug interactions. Bedaquiline, pretomanid, and linezolid were recently found to be more potent in treating mice infected with the Mtb HN878 strain than the H37Rv strain (80). When combined, the drugs antagonized each other for treating Mtb strain HN878-infected mice. Despite this antagonism, the BPaL combination was 
highly effective at curing mice infected with either Mtb strain. These in vivo results are consistent with our findings that $\mathrm{BPaL}$ is a highly potent but antagonistic drug combination for in vitro treatment of Mtb Erdman. One view of how drugs in combination exert their effect on cell populations is that each drug targets a different subpopulation rather than multiple drugs targeting the same cells $(73,79)$. Drug interactions would then explain how well a drug acts on the cellular population that was not susceptible to the other drugs in the multidrug treatment. This leads to the hypothesis that very potent drugs that alone can kill most of the cells in a population would achieve high maximum effect when combined but may tend toward antagonism rather than synergy. Study of the multidrug anticancer therapy R-CHOP (Table 1) supports this hypothesis (73), and an expanded study using more antibiotics could be used to test this hypothesis in tuberculosis. Our study suggests that for TB, potent drug combinations should be prioritized for further study and should not necessarily be deprioritized if they are antagonistic in in vitro assays.

Our approach enabled us to determine the relative importance of specific in vitro models to predict outcomes in mice, thereby serving to validate which growth conditions map to in vivo responses. We note that signatures including several in vitro models perform better than signatures using data from only one in vitro model, perhaps because the lesion microenvironment is complex and constitutes multiple stressors that affect Mtb drug response. It may also indicate that none of the physiological states imposed by the in vitro models we used in this study was so dominant that it drives Mtb in vivo drug treatment response. We chose several lipids to serve as carbon sources in our in vitro models because of the important role of lipids and cholesterol specifically for Mtb growth, survival, and infectivity (81-90). The cholesterol in vitro model was the top-performing single in vitro model classifier for the RMM outcome and performed almost as well as the classifier with all in vitro models. This is consistent with the importance of cholesterol metabolism for Mtb 
survival and infectivity $(24,85-87)$. We also observed that other lipid-rich environments (modeled by using short-chain fatty acids butyrate or valerate as carbon sources) induced distinct drug response patterns and that the best classifiers for both RMM and $\mathrm{BHeB}$ outcomes utilized metrics from multiple lipid-rich growth conditions. These findings suggest that measuring drug combination responses with a suite of simple growth environments may be sufficient to model the complex lipid environment encountered in TB lesions.

Mtb in the RMM mouse strains are thought to be primarily intracellular (24), and intracellular Mtb are exposed to the acidification of the phagolysosome $(36,37)$. Therefore, we expected the acidic growth environment to be a driver in classifiers for the RMM. We found that measurements from the acidic growth environment alone were not strongly predictive of outcomes in the RMM but that these metrics were prominent in the best mixed-condition classifiers. Furthermore, other single growth environment models perform better than the acidic model (Table S6). These results indicate that response to acidic stress is important for Mtb intracellular survival to drug treatment in the RMM, but adaptation to other environmental factors (such as lipid carbon sources) are important drivers of treatment response. We also observed that the acidic model was prominent among the best classifiers for the bactericidal outcome in the $\mathrm{C} 3 \mathrm{HeB} / \mathrm{FeJ}$ mouse strain (BHeB outcome). The $\mathrm{C} 3 \mathrm{HeB} / \mathrm{FeJ}$ mice are noted for the formation of the caseous necrotic granulomas (type II lesions, (76)) that have been shown to have a neutral $\mathrm{pH}(\mathrm{pH}>7)(91)$ and with primarily extracellular Mtb (76). However, these animals have abundant intracellular bacteria in other lesion types and within macrophages that acidify the intracellular Mtb compartments (77), which may explain why acidic growth environments are important predictors of drug response in this mouse model.

The microenvironments in TB granulomas are complex, yet we were able to combine measurement in a "sum-of-parts" approach from relatively simple growth environments to model treatment 
outcomes (Fig. 4B, Figure S8). These results indicate that there is predictive drug combination response information obtained from simple in vitro models that only needs to be combined correctly to predict drug treatment outcomes in mice. The practical implication is that researchers can choose a subset of the most amenable in vitro models for performing drug combination experiments and still retain predictive capacity. We successfully model RMM and BHeB outcomes using this compendium but not the BMM outcome, suggesting that there may be other important factors or emergent properties of complex environments encountered during infection that were not included in our measurement set. These factors may include other carbon sources, nutrient availability, iron limitation, oxygen tension, human serum, or other entry mechanisms into dormancy (e.g., via different lipids or other combinations of stressors) (91-93). Macrophage activation status has also been linked to Mtb drug susceptibility (94), and therefore models of cytokine-induced activation of macrophages may be more relevant to specific in vivo outcomes. Future studies measuring drug combination response in other and more complex in vitro models may permit accurate modeling of the BMM outcome and improve the accuracy of predictions of outcomes in RMM, BHeB.

We anticipate that our in vitro-to-in vivo pipeline for drug combination predictions may be applied to study treatment outcomes in other animals and clinical studies. Predictions using our RMM classifiers suggest the potential for using DiaMOND data to model responses in the clinic. The moxifloxacin containing regimens, HRZM and MRZE, were expected to shorten treatment time in humans by two months based on preclinical mouse studies but did not show non-inferiority to HRZE in the ReMOX clinical trial (95). A meta-analysis of mouse relapse outcome studies after the trial completion revealed that the treatment shortening of these combinations was expected to be smaller than initially thought and that perhaps could explain the ReMOX trial results (62). A 
recent PET/CT study in humans of 14-day treatment regimens also found MRZE and HRZE to be comparable in the reduction of lesion volume (96). The consensus RMM classifier predictions from our study predicted that the MRZE and HRZM combinations were not likely to show treatment improvement ( $47 \%$ and $53 \%$ chance of being $\mathrm{C} 1$, respectively), consistent with the ReMOX trial outcome, PET/CT study, and mouse meta-analysis. Based on our prediction probabilities, MRZE and HRZM would not have been prioritized for further study compared to other combinations, like $\mathrm{BPaMZ}$ ( $83 \%$ chance of being $\mathrm{C} 1)$, that are being evaluated in ongoing clinical trials (97). Another example of the potential utility of the DiaMOND compendium for clinical predictions comes from the "Study 31" clinical trial. The preliminary results from "Study 31" show treatment shortening of the continuation phase using PHZM compared with the standard treatment of HRZE (intensive) followed by HR (continuation) (9). A third treatment arm (PHZE) did not show improvement compared with the standard treatment. Similar to our ReMOX predictions, our consensus prediction indicated PHZE to have a low probability for treatment improvement over standard of care $(42 \%$ chance of being $\mathrm{C} 1)$ despite the mouse outcome indicating C1 classification. The ReMOX and "Study 31" examples suggest that the DiaMOND compendium contains information that is relevant to understanding clinical outcomes while contradictory to the mouse outcomes. Together these results indicate that the DiaMOND compendium could be used in future modeling and clinical outcomes predictions $(98,99)$.

Several changes to the experimental design may improve this pipeline. The importance of potency metrics in signatures of combination efficacy is perhaps surprising given that we design combination dose responses to have equipotent combinations of each drug. There is growing evidence that there is differential drug penetration into the lesions where Mtb is found (78), which would lead to non-equipotent levels of drug reaching Mtb cells. Utilizing pharmacokinetic data to 
design drug combinations may increase this approach's utility and power and lead to a more predictive DiaMOND compendium dataset. The current standard of care and other new regimens (e.g., "Study 31" and SimpliciTB) involve intensive and continuation phases of treatment. Including sequential treatments in an experimental approach could help understand how prior treatment sensitizes the bacterial population to future treatment regimens. One reason to use combination therapy for TB is to slow the acquisition of drug resistance. A systematic study of the drug combination space in different growth environments can also be used to investigate the evolution of drug resistance. For example, antagonistic drug interactions have been shown to suppress the evolution of drug resistance $(56,73,100,101)$, and the evolution of drug resistance can be tied to the growth rate and duration of drug exposure $(56,80,102,103)$. Finally, the depth of the DiaMOND compendium may be well-complemented with transcriptomic data of drug response to prioritize drug combinations based on predicted mechanisms of drug interaction (104).

TB is not the only disease that benefits from combination therapy. We expect that our pipeline may be adapted and applied to optimize multidrug regimens for other diseases, including cancers, HIV/AIDs, and multi-drug resistant bacterial pathogens. Beyond our use of the TB DiaMOND compendium to describe combination efficacies in mouse models, we anticipate that this dataset may be used for other systematic studies of drug combination response.

\section{Materials and Methods}

\section{Strains and media}

M. tuberculosis Erdman strain was transformed with pMV306hsp+LuxG13 to generate an autoluminescent strain that was used for all experiments in this study (Addgene plasmid \# 26161; 
http://n2t.net/addgene:26161; RRID:Addgene_26161) (105), see Supplemental Materials). We used the mouse cell line, J774 as a model of intracellular residency because J774 cells have been used as a macrophage-like cell line to study early infection processes and Mtb drug response to complex host-like intracellular environment $(48,106)$.

Standard 7H9 Middlebrook medium supplemented with $0.2 \%$ glycerol, $10 \%$ OADC $(0.5 \mathrm{~g} / \mathrm{L}$ oleic acid, $50 \mathrm{~g} / \mathrm{L}$ albumin, $20 \mathrm{~g} / \mathrm{L}$ dextrose and $0.04 \mathrm{~g} / \mathrm{L}$ catalase) and $0.05 \%$ Tween- 80 with $25 \mu \mathrm{g} / \mathrm{mL}$ kanamycin was used for Mtb strain maintenance. Growth and culturing were performed at $37^{\circ} \mathrm{C}$ with aeration unless noted. All in vitro model media were buffered with $100 \mathrm{mM} 3-(\mathrm{N}-$ morpholino)propanesulfonic acid (MOPS, $\mathrm{pH}$ 7), unless noted, and filter-sterilized prior to use. The acidic model was based on the standard 7H9 Middlebrook media above and buffered with 100 mM 2-(N-morpholino)ethanesulfonic acid (MES) to $\mathrm{pH}$ 5.7. For acclimation to lipid carbon sources, a base medium consisting of $7 \mathrm{H} 9$ powder $(4.7 \mathrm{~g} / \mathrm{L})$, fatty acid-free BSA $(0.5 \mathrm{~g} / \mathrm{L}), \mathrm{NaCl}$ (100mM) and tyloxapol $(0.05 \%)$ with $25 \mu \mathrm{g} / \mathrm{mL}$ kanamycin was used and the lipids sodium butyrate $(5 \mathrm{mM}$, final concentration), valeric acid $(0.1 \%$ final concentration $)$ or cholesterol $(0.05 \mathrm{mM}$ or $0.2 \mathrm{mM}$ final concentration) were added to the base medium. For the cholesterol media, a cholesterol stock solution $(100 \mathrm{mM})$ was first prepared by dissolving cholesterol in a 1:1 (v/v) mixture of ethanol and tyloxapol and heated to $80^{\circ} \mathrm{C}$ for 30 minutes and added to pre-warmed $\left(37^{\circ} \mathrm{C}\right)$ base medium $(32)$. The dormancy media was based on the butyrate media with the addition of sodium nitrate $(5 \mathrm{mM})$ as a terminal electron acceptor $(38,41-43)$. $\mathrm{J} 774$ cells were cultured as previously described (106). Briefly, J774 cells were cultured in high glucose DMEM supplemented with $2 \mathrm{mM}$ L-glutamine, $1 \mathrm{mM}$ sodium pyruvate, and $10 \%$ heat-inactivated fetal bovine serum $(\mathrm{FBS})$ at $37^{\circ} \mathrm{C}$ in $5 \% \mathrm{CO} 2$. Media was changed every one-three days and cells passaged at $\sim 80 \%$ confluence. Standard 7 H10 Middlebrook agar plates supplemented with $0.5 \%$ 
glycerol, $10 \%$ OADC, $0.05 \%$ Tween-80 and $25 \mu \mathrm{g} / \mathrm{mL}$ kanamycin were used for enumerating colonies.

\section{Mtb in vitro model acclimation}

Mtb were inoculated into standard 7H9 Middlebrook medium, grown to mid-log phase (optical density, $\mathrm{OD}_{600} \sim 0.5-0.7$ ) and were subcultured for less than two weeks prior to acclimation to assay medium. For acclimation to standard and acidic media, Mtb cells were diluted into assay media at a starting density of $\mathrm{OD}_{600}=0.05$, acclimated for 3-5 doubling times or until they reached mid-log phase $\left(\mathrm{OD}_{600} \sim 0.5-0.7\right)$, diluted to $\mathrm{OD}_{600}=0.05$ and grown back to mid-log phase before use in DiaMOND assays.

Similar to standard, and acidic conditions, Mtb were acclimated to butyrate, and valerate media and acclimated cells were frozen for use in assays. Frozen acclimated Mtb in butyrate and valerate media were inoculated into assay media, grown to mid-log phase $\left(\mathrm{OD}_{600} \sim 0.5-0.7\right)$, diluted into fresh lipid media at a starting concentration of $\mathrm{OD}_{600}=0.05$ and grown back to mid-log phase $\left(\mathrm{OD}_{600} \sim 0.5-0.7\right)$ and used for DiaMOND assays. The dormancy model used Mtb acclimated to butyrate medium grown to mid-log phase $\left(\mathrm{OD}_{600} \sim 0.5-0.7\right)$ and then diluted to a starting $\mathrm{OD}_{600}$ 0.05 in dormancy media. For the dormancy model (d), cells were incubated at $37^{\circ} \mathrm{C}$ without aeration for 28 days, which reduced autoluminescence close to media-only background levels, which we interpret as being dormant with very low metabolic activity.

Mtb growth on cholesterol media slowed without the exchange of fresh medium. Cholesterol and cholesterol-high acclimation were similar to standard and acidic conditions with fresh media exchanges every seven days to ensure continued growth. Mtb acclimated between 14 and 28 days were used for assays. Mtb growth rate on cholesterol-high was faster (four day doubling time) than cholesterol (seven day doubling time). 
For the intracellular model, J774 cells were plated at 375,000 cells $/ \mathrm{mL}$ in $384-$ well plates and cultured overnight, expecting one doubling prior to infection. Mtb grown to mid-log phase in standard media was syringe-passed 8 times with a 25-gauge needle to reach a single-cell suspension, and J774s were infected with Mtb at MOI 2 for 24 hours followed by drug treatment for 5 days.

Drugs, dose responses, and dispensing

The drugs used in this study are listed in Table 1. All drugs were reconstituted and diluted in DMSO except for PZA for the intracellular model; to avoid exceeding the DMSO limit (0.5\%) in the intracellular condition, PZA was diluted in $1 \mathrm{x}$ phosphate-buffered saline with $0.01 \%$ TritonX. Drugs were dispensed with an HP D300e digital dispenser, and locations were randomized to reduce plate effects. For each in vitro model, the concentration to achieve $90 \%$ inhibition (IC90) was determined. $\mathrm{IC}_{90}$ were used to design combination dose responses with equipotent mixtures of drugs (31). A ten-dose resolution with 1.5- or 2-fold dose spacing was used for all experiments.

\section{Treatment and DiaMOND assays}

Mtb were acclimated to in vitro model media prior to drug treatment as described above. For acidic, butyrate, cholesterol, cholesterol-high, standard, and valerate models: $50 \mu \mathrm{L}$ of acclimated Mtb at the indicated density was added to each well in 384-well plates containing freshly dispensed drugs and incubated at $37^{\circ} \mathrm{C}$ in humidified bags to prevent evaporation. Edge wells contained media but were not used for assays. For the dormancy model: Mtb were acclimated as described above, gently resuspended, and $20 \mu \mathrm{L}$ of dormant Mtb culture was transferred to each well on the assay plates. Plates were sealed with PCR seals to reduce oxygen exposure during drug treatment and incubated for seven days. We measured regrowth after drug treatment as a readout of drug effect during dormancy. Therefore, after drug treatment, plate seals were removed, $80 \mu \mathrm{L}$ of standard media was 
added to each well, and plates were incubated at $37^{\circ} \mathrm{C}$ in humidified bags to prevent evaporation. For the intracellular model: drugs were printed into media-only plates and transferred onto infected J774 cells 24 hours after Mtb infection. To accommodate quality control assessment, we included multiple untreated and positive drug treatment controls in each plate as well as uninfected J774 cells for the intracellular model. (See Supplementary Materials).

\section{Plate measurements}

Luminescence and $\mathrm{OD}_{600}$ measurements were made at 3-5 time points per sample on a Synergy Neo2 Hybrid Multi-Mode Reader. Time points were based on the approximate doubling time of each model. To simplify the analysis, we generally compare time points at either a relatively similar time point (constant) or time $\sim 4-5 \mathrm{x}$ doubling times after drug exposure (terminal time point). Constant and terminal time points correspond to the same set of measurements for the standard and intracellular in vitro models (constant/terminal, CT). For the dormancy model, plate readings were made during recovery in standard media, and time points were selected based on doubling time in standard media. For the dormancy and intracellular models, $\mathrm{OD}_{600}$ measurements could not reflect Mtb biomass alone, so only luminescence measurements are used. Autoluminescence has been demonstrated as a proxy for Mtb cell growth (105) and viability in response to drug treatment $(107,108)$. To benchmark changes in luminescence to changes in growth in our conditions, we performed a series of drug treatment experiments in the dormancy and intracellular models (see Supplementary Materials and Fig. S1). Briefly, cells were treated as described above, followed by plating treated cells on 7H10 plates to enumerate colony forming units (CFU). Portions of the luminescence dose response curve that correlated with CFU changes were considered indicative of growth inhibition, and metrics derived from these portions of the curve were used for analysis. 


\section{Data processing and metric calculation}

Data processing and dose response metric calculation were performed using custom MATLAB scripts. In brief, raw data were background-subtracted using the median of media-only wells and normalized to the mean of untreated wells within each plate. For the intracellular model, uninfected macrophages provided the background (rather than media only) for subtraction from raw data, and subsequently, data was normalized to (infected) untreated within each plate. A 3-parameter Hill function was fit to each dose response (single drug or combination). Inhibitory concentrations (ICs) were calculated based on the Hill curve parameters. The area under the curve at $25 \%$ inhibition $\left(\mathrm{AUC}_{25}\right)$ was calculated using the integral of the fit curves from 0 to the $25 \%$ inhibitory concentration $\left(\mathrm{IC}_{25}\right)$ and normalized to the $\mathrm{IC}_{25}$, allowing comparisons between drug combinations. Drug interaction scores were quantified by the fractional inhibitory concentration (FIC) using Loewe additivity and Bliss independence (See Box). FICs calculated by Loewe additivity and Bliss independence were well correlated, and neither model was observed to suffer from significant bias relative to the order of the drug combination (109) (Supplementary Material); therefore, we proceeded to analyze drug interactions based on Loewe additivity. The growth rate inhibition (GR) metrics were calculated as previously described (55). See Supplementary Materials for details on data processing and analysis.

\section{Data quality}

Experiments were performed in a minimum of biological triplicate. Comparisons of data between plates and between experimental days required data quality control assessment. Each dose response was assigned a quality score that takes into account the overall quality of the data from a plate, the quality of fit of the Hill function, the single drug dose responsiveness from an experiment, and in the case of drug combinations, the equipotency in the drug combination dose 
responses (Supplementary Materials). In brief, plate data quality was assessed with a Z'-score using multiple untreated (negative) and complete inhibition treatment (positive) wells in each plate. The fitting of the Hill function was assessed by the coefficient of determination $\left(\mathrm{R}^{2}\right)$ of the fit as well as the closeness of the $\mathrm{E}_{\text {inf }}$ for each fit to the maximum observed effect for each dose response curve. Drug combination equipotency was assessed by comparing the proportional combinations normalized to their respective MICs and the idealized combination of drugs if they were perfectly equipotent. Dose responses with poor quality scores were excluded from further analysis.

\section{Computational Analyses}

Biological replicate dose response and drug interaction data passing quality control were averaged. Means of replicate data were used for all downstream analyses unless noted. Hierarchical clustering was performed using cosine distance, and heatmaps with complete linkage dendrograms were generated using MATLAB. Other data preparation and visualizations were performed in R studio (version 3.5.3) using the tidyverse environment packages and ggplot2 and ggpubr packages for visualization. Data table import and export were performed in R using the openxl and readxls packages

PCA was performed in $\mathrm{R}$ using the prcomp function from the stats package with each feature scaled to have unit variance before PCA. Some features were missing data; e.g., FIC 90 metrics were missing because single drugs did not achieve $\mathrm{IC}_{90}$. Features with more than $35 \%$ missing data points were excluded from PCA. The remaining missing values were imputed using the mean of the corresponding input features (mean imputation) (110).

\section{Machine learning}


The machine learning in $\mathrm{R}$ (mlr v2.17.0) package was used for all machine learning tasks involving projections of the original features onto the principal component (PC) space as the input features and classified drug combination outcome $(\mathrm{C} 0$ or $\mathrm{C} 1)$ as labels.

Feature selection, feature number optimization, and model validation

The Kruskal-Wallis test was used to rank order the PC input features for ML based on the ability to discriminate outcome classes $\mathrm{C} 0$ and $\mathrm{C} 1$. As there were a limited number of drug combinations, we aimed to reduce the number of features used in the model. A Monte-Carlo resampling strategy was used to split the training data into $70 / 30 \%$ training/test partitions, to which we applied grid search to find the number of features that produced the largest test AUC. This feature number optimization was repeated five times for each training set, and the smallest feature set from the five iterations was chosen as the final training feature set. Models were trained on the full set of training data, and performance on new data was estimated using standard 5-fold cross validation. Validation was performed by projecting new data onto the PC space used for the model training and testing model classification performance.

\section{Machine learner packages}

Upon feature selection, machine learning algorithms were compared using standard 5-fold cross validation. The performance was evaluated using the AUC and the F-score (F1). The mlr package made possible on-demand loading of learners from other $\mathrm{R}$ packages, including Bayesian additive regression tree (bartMachine, v1.2.5.1), random forest (randomForestSRC, v2.9.3), extreme gradient boosting (xgboost, v1.1.1.1), logistic regression (stats), naive bayes (e1071, v1.7-3), support vector machine (e1071, v1.7-3), and weighted k-nearest neighbors (kknn, v1.3.1).

\section{Statistical Analysis}


Differences between outcome class groups for DiaMOND features or PCs were assessed by means

(IC90 averages), medians (class comparisons), and standard deviation of drug combinations from each outcome group in each in vitro model. Because data normality could not easily be assessed with small numbers of drug combinations in each group, the Wilcoxon rank-sum test was used to compare outcome group means for statistical significance. Student's t-tests were used for testing hypotheses of differences between model performance distributions. The hypothesis that Loewe and Bliss interaction (FIC) scores were correlated was tested using Pearson correlations. Statistical analyses were performed using the stats, ggpubr, or the rstatrix packages in R version 3.5.3.

\section{Supplementary Materials}

Materials and Methods

Fig. S1. Benchmarking cell viability with luminescence measurements.

Fig. S2. Comparing null reference models for drug interaction scoring.

Fig. S3. Intermediate potency drug interaction profiles.

Fig. S4. Alternative potency metric profiles for selected drug combinations.

Fig. S5. Alternative potency metric profiles for DiaMOND compendium.

Fig. S6. Outcome class separation in single in vitro model principal component analyses (PCAs).

Fig. S7. BMM classifier performance on training and test data.

Fig. S8. BHeB in vitro model subset model performance distributions.

Fig. S9. BHeB in vitro model with and without dormancy model performance distributions.

Table S1. Experiment time points and estimated growth amounts for in vitro models. 
Table S2. Drug information table.

Table S3. Drug $\mathrm{IC}_{90}$ for in vitro models.

Table S4. Drug combinations with RMM outcomes for 2- and 3-way drug combinations.

Table S5. RMM PC class separation.

Table S6. Machine learning algorithm benchmarking performance metrics.

Table S7. RMM model validation set.

Table S8. RMM single in vitro model classifier performance.

Table S9. Drug combinations with BMM outcomes for 1-, 2- and 3-way drug combinations.

Table S10. Drug combinations with BHeB outcomes for 1-, 2- and 3-way drug combinations.

Table S11. BHeB PC class separation.

\section{References and Notes:}

1. "Global tuberculosis report 2020," (World Health Organization, Geneva, 2020). https://apps.who.int/iris/handle/10665/336069

2. "The End TB Strategy," (World Health Organization, Geneva, 2014). https://www.who.int/tb/strategy/End_TB_Strategy.pdf?ua=1

3. W. Fox, G. A. Ellard, D. A. Mitchison, Studies on the treatment of tuberculosis undertaken by the British Medical Research Council Tuberculosis Units, 1946-1986, with relevant subsequent publications. The International Journal of Tuberculosis and Lung Disease 3, S231-S279 (1999). 
4. C. A. Kerantzas, W. R. Jacobs, Origins of Combination Therapy for Tuberculosis:

Lessons for Future Antimicrobial Development and Application. mBio 8, e01586-01516 (2017).

5. S. Tiberi, N. du Plessis, G. Walzl, M. J. Vjecha, M. Rao, F. Ntoumi, S. Mfinanga, N. Kapata, P. Mwaba, T. D. McHugh, G. Ippolito, G. B. Migliori, M. J. Maeurer, A. Zumla, Tuberculosis: progress and advances in development of new drugs, treatment regimens, and host-directed therapies. The Lancet Infectious Diseases 18, e183-e198 (2018).

6. D. A. Mitchison, Modern methods for assessing the drugs used in the chemotherapy of mycobacterial disease. Journal of Applied Bacteriology 81, 72S-80S (1996).

7. J. C. Evans, V. Mizrahi, Priming the tuberculosis drug pipeline: new antimycobacterial targets and agents. Curr Opin Microbiol 45, 39-46 (2018).

8. S. E. Dorman, P. Nahid, E. V. Kurbatova, S. V. Goldberg, L. Bozeman, W. J. Burman, K. C. Chang, M. Chen, M. Cotton, K. E. Dooley, M. Engle, P. J. Feng, C. V. Fletcher, P. Ha, C. M. Heilig, J. L. Johnson, E. Lessem, B. Metchock, J. M. Miro, N. V. Nhung, A. C. Pettit, P. P. J. Phillips, A. T. Podany, A. E. Purfield, K. Robergeau, W. Samaneka, N. A. Scott, E. Sizemore, A. Vernon, M. Weiner, S. Swindells, R. E. Chaisson, High-dose rifapentine with or without moxifloxacin for shortening treatment of pulmonary tuberculosis: Study protocol for TBTC study 31/ACTG A5349 phase 3 clinical trial. Contemp Clin Trials 90, 105938 (2020).

9. CDC, Media Statement: Landmark TB Trial Identifies Shorter-Course Treatment Regimen | CDC, (CDC, 2020). https://www.cdc.gov/nchhstp/newsroom/2020/landmarktb-trial-media-statement.html 
10. A. Iacobino, G. Piccaro, F. Giannoni, A. Mustazzolu, L. Fattorini, Fighting tuberculosis by drugs targeting nonreplicating $<\mathrm{i}>$ Mycobacterium tuberculosis $</ \mathrm{i}>$ bacilli.

International Journal of Mycobacteriology 6, 213-221 (2017).

11. Y. Liu, M. Matsumoto, H. Ishida, K. Ohguro, M. Yoshitake, R. Gupta, L. Geiter, J. Hafkin, Delamanid: From discovery to its use for pulmonary multidrug-resistant tuberculosis (MDR-TB). Tuberculosis 111, 20-30 (2018).

12. J. van den Boogaard, G. S. Kibiki, E. R. Kisanga, M. J. Boeree, R. E. Aarnoutse, New Drugs against Tuberculosis: Problems, Progress, and Evaluation of Agents in Clinical Development. Antimicrobial Agents and Chemotherapy 53, 849-862 (2009).

13. F. Conradie, A. H. Diacon, N. Ngubane, P. Howell, D. Everitt, A. M. Crook, C. M. Mendel, E. Egizi, J. Moreira, J. Timm, T. D. McHugh, G. H. Wills, A. Bateson, R. Hunt, C. Van Niekerk, M. Li, M. Olugbosi, M. Spigelman, Treatment of Highly Drug-Resistant Pulmonary Tuberculosis. New England Journal of Medicine 382, 893-902 (2020).

14. E. Pontali, R. Centis, L. D'Ambrosio, F. Toscanini, G. B. Migliori, Recent evidence on delamanid use for rifampicin-resistant tuberculosis. J Thorac Dis 11, S457-S460 (2019).

15. clinicaltrials.gov. National Library of Medicine. (2011). NCT01215851. Evaluation of Early Bactericidal Activity in Pulmonary Tuberculosis With(J-M-Pa-Z) (NC-001). https://ClinicalTrials.gov/show/NCT01215851

16. clinicaltrials.gov. National Library of Medicine. (2013). NCT01498419. Evaluation of 8 Weeks of Treatment With the Combination of Moxifloxacin, PA-824 and Pyrazinamide in Patients With Drug Sensitive and Multi Drug-Resistant Pulmonary Tuberculosis (TB) (NC-002). https://ClinicalTrials.gov/show/NCT01498419 
17. clinicaltrials.gov. National Library of Medicine. (2018). NCT02342886. Shortening Treatment by Advancing Novel Drugs. https://ClinicalTrials.gov/show/NCT02342886

18. clinicaltrials.gov. National Library of Medicine. (2016). NCT02193776. A Phase 2 to Evaluate the Efficacy, Safety and Tolerability of Combinations of Bedaquiline, Moxifloxacin, PA-824 and Pyrazinamide in Adult Subjects With Drug-Sensitive or Multi Drug-Resistant Pulmonary Tuberculosis. https://ClinicalTrials.gov/show/NCT02193776

19. clinicaltrials.gov. National Library of Medicine. (2020). NCT02410772. TBTC Study 31: Rifapentine-containing Tuberculosis Treatment Shortening Regimens. https://ClinicalTrials.gov/show/NCT02410772

20. K. E. Dooley, P. P. J. Phillips, P. Nahid, M. Hoelscher, Challenges in the clinical assessment of novel tuberculosis drugs. Advanced Drug Delivery Reviews 102, 116-122 (2016).

21. T. Gumbo, A. J. Lenaerts, D. Hanna, K. Romero, E. Nuermberger, Nonclinical Models for Antituberculosis Drug Development: A Landscape Analysis. The Journal of Infectious Diseases 211, S83-S95 (2015).

22. E. Nuermberger, S. Tyagi, R. Tasneen, K. N. Williams, D. Almeida, I. Rosenthal, J. H. Grosset, Powerful Bactericidal and Sterilizing Activity of a Regimen Containing PA-824, Moxifloxacin, and Pyrazinamide in a Murine Model of Tuberculosis. Antimicrobial Agents and Chemotherapy 52, 1522-1524 (2008).

23. R. Tasneen, S. Y. Li, C. A. Peloquin, D. Taylor, K. N. Williams, K. Andries, K. E. Mdluli, E. L. Nuermberger, Sterilizing activity of novel TMC207- and PA-824containing regimens in a murine model of tuberculosis. Antimicrob Agents Chemother 55, 5485-5492 (2011). 
24. E. L. Nuermberger, Preclinical Efficacy Testing of New Drug Candidates. Microbiology Spectrum 5, (2017).

25. A. Apt, I. Kramnik, Man and mouse TB: contradictions and solutions. Tuberculosis (Edinb) 89, 195-198 (2009).

26. I. Kramnik, G. Beamer, Mouse models of human TB pathology: roles in the analysis of necrosis and the development of host-directed therapies. Semin Immunopathol 38, 221237 (2016).

27. E. R. Driver, G. J. Ryan, D. R. Hoff, S. M. Irwin, R. J. Basaraba, I. Kramnik, A. J. Lenaerts, Evaluation of a mouse model of necrotic granuloma formation using $\mathrm{C} 3 \mathrm{HeB} / \mathrm{FeJ}$ mice for testing of drugs against Mycobacterium tuberculosis. Antimicrob Agents Chemother 56, 3181-3195 (2012).

28. J. P. Lanoix, A. J. Lenaerts, E. L. Nuermberger, Heterogeneous disease progression and treatment response in a $\mathrm{C} 3 \mathrm{HeB} / \mathrm{FeJ}$ mouse model of tuberculosis. Dis Model Mech $\mathbf{8}$, 603-610 (2015).

29. A. Lenaerts, C. E. Barry, 3rd, V. Dartois, Heterogeneity in tuberculosis pathology, microenvironments and therapeutic responses. Immunol Rev 264, 288-307 (2015).

30. T. Parish, In vitro drug discovery models for Mycobacterium tuberculosis relevant for host infection. Expert Opin Drug Discov 15, 349-358 (2020).

31. M. Cokol, N. Kuru, E. Bicak, J. Larkins-Ford, B. B. Aldridge, Efficient measurement and factorization of high-order drug interactions in $<$ em $>$ Mycobacterium tuberculosis $</$ em $>$. Science Advances 3, e1701881 (2017). 
32. W. Lee, B. C. VanderVen, R. J. Fahey, D. G. Russell, Intracellular Mycobacterium tuberculosis exploits host-derived fatty acids to limit metabolic stress. J Biol Chem 288, 6788-6800 (2013).

33. J. V. Early, A. Casey, M. A. Martinez-Grau, I. C. Gonzalez Valcarcel, M. Vieth, J. Ollinger, M. A. Bailey, T. Alling, M. Files, Y. Ovechkina, T. Parish, Oxadiazoles Have Butyrate-Specific Conditional Activity against Mycobacterium tuberculosis. Antimicrob Agents Chemother 60, 3608-3616 (2016).

34. K. Pethe, P. C. Sequeira, S. Agarwalla, K. Rhee, K. Kuhen, W. Y. Phong, V. Patel, D. Beer, J. R. Walker, J. Duraiswamy, J. Jiricek, T. H. Keller, A. Chatterjee, M. P. Tan, M. Ujjini, S. P. Rao, L. Camacho, P. Bifani, P. A. Mak, I. Ma, S. W. Barnes, Z. Chen, D. Plouffe, P. Thayalan, S. H. Ng, M. Au, B. H. Lee, B. H. Tan, S. Ravindran, M. Nanjundappa, X. Lin, A. Goh, S. B. Lakshminarayana, C. Shoen, M. Cynamon, B. Kreiswirth, V. Dartois, E. C. Peters, R. Glynne, S. Brenner, T. Dick, A chemical genetic screen in Mycobacterium tuberculosis identifies carbon-source-dependent growth inhibitors devoid of in vivo efficacy. Nat Commun 1, 57 (2010).

35. V. Guerrini, B. Prideaux, L. Blanc, N. Bruiners, R. Arrigucci, S. Singh, H. P. Ho-Liang, H. Salamon, P. Y. Chen, K. Lakehal, S. Subbian, P. O'Brien, L. E. Via, C. E. Barry, 3rd, V. Dartois, M. L. Gennaro, Storage lipid studies in tuberculosis reveal that foam cell biogenesis is disease-specific. PLoS Pathog 14, e1007223 (2018).

36. J. J. Baker, S. J. Dechow, R. B. Abramovitch, Acid Fasting: Modulation of Mycobacterium tuberculosis Metabolism at Acidic pH. Trends Microbiol 27, 942-953 (2019). 
37. O. H. Vandal, C. F. Nathan, S. Ehrt, Acid resistance in Mycobacterium tuberculosis. $J$ Bacteriol 191, 4714-4721 (2009).

38. B. Gold, C. Nathan, Targeting Phenotypically Tolerant Mycobacterium tuberculosis. Microbiol Spectr 5, (2017).

39. C. de Miranda Silva, A. Hajihosseini, J. Myrick, J. Nole, A. Louie, S. Schmidt, G. L. Drusano, Effect of Moxifloxacin plus Pretomanid against Mycobacterium tuberculosis in Log Phase, Acid Phase, and Nonreplicating-Persister Phase in an In Vitro Assay. Antimicrob Agents Chemother 63, (2019).

40. G. L. Drusano, S. Kim, M. Almoslem, S. Schmidt, D. Z. D'Argenio, J. Myrick, B. Duncanson, J. Nole, D. Brown, C. A. Peloquin, M. Neely, W. Yamada, A. Louie, The Funnel: a Screening Technique for Identifying Optimal Two-Drug Combination Chemotherapy Regimens. Antimicrob Agents Chemother 65, (2021).

41. A. Cunningham-Bussel, F. C. Bange, C. F. Nathan, Nitrite impacts the survival of Mycobacterium tuberculosis in response to isoniazid and hydrogen peroxide. Microbiologyopen 2, 901-911 (2013).

42. A. Cunningham-Bussel, T. Zhang, C. F. Nathan, Nitrite produced by Mycobacterium tuberculosis in human macrophages in physiologic oxygen impacts bacterial ATP consumption and gene expression. Proc Natl Acad Sci U S A 110, E4256-E4265 (2013).

43. C. D. Sohaskey, Nitrate enhances the survival of Mycobacterium tuberculosis during inhibition of respiration. Journal of bacteriology 190, 2981-2986 (2008).

44. D. F. Warner, V. Mizrahi, Tuberculosis chemotherapy: the influence of bacillary stress and damage response pathways on drug efficacy. Clin Microbiol Rev 19, 558-570 (2006). 
45. Y. Zhang, W. W. Yew, M. R. Barer, Targeting Persisters for Tuberculosis Control. Antimicrobial Agents and Chemotherapy 56, 2223-2230 (2012).

46. E. A. Lamont, N. A. Dillon, A. D. Baughn, The Bewildering Antitubercular Action of Pyrazinamide. Microbiol Mol Biol Rev 84, (2020).

47. E. A. Lamont, A. D. Baughn, Impact of the host environment on the antitubercular action of pyrazinamide. EBioMedicine 49, 374-380 (2019).

48. D. Pires, E. Valente, M. F. Simoes, N. Carmo, B. Testa, L. Constantino, E. Anes, Esters of Pyrazinoic Acid Are Active against Pyrazinamide-Resistant Strains of Mycobacterium tuberculosis and Other Naturally Resistant Mycobacteria In Vitro and Ex Vivo within Macrophages. Antimicrob Agents Chemother 59, 7693-7699 (2015).

49. K. Rohde, R. M. Yates, G. E. Purdy, D. G. Russell, Mycobacterium tuberculosis and the environment within the phagosome. Immunol Rev 219, 37-54 (2007).

50. O. Alfarisi, W. A. Alghamdi, M. H. Al-Shaer, K. E. Dooley, C. A. Peloquin, Rifampin vs. rifapentine: what is the preferred rifamycin for tuberculosis? Expert Rev Clin Pharmacol 10, 1027-1036 (2017).

51. A. Koul, L. Vranckx, N. Dhar, H. W. H. Göhlmann, E. Özdemir, J.-M. Neefs, M. Schulz, P. Lu, E. Mørtz, J. D. McKinney, K. Andries, D. Bald, Delayed bactericidal response of Mycobacterium tuberculosis to bedaquiline involves remodelling of bacterial metabolism. Nature Communications 5, 3369 (2014).

52. Z. Xie, N. Siddiqi, E. J. Rubin, Differential antibiotic susceptibilities of starved Mycobacterium tuberculosis isolates. Antimicrob Agents Chemother 49, 4778-4780 (2005). 
53. J. C. Betts, P. T. Lukey, L. C. Robb, R. A. McAdam, K. Duncan, Evaluation of a nutrient starvation model of Mycobacterium tuberculosis persistence by gene and protein expression profiling. Mol Microbiol 43, 717-731 (2002).

54. L. G. Wayne, H. A. Sramek, Metronidazole is bactericidal to dormant cells of Mycobacterium tuberculosis. Antimicrob Agents Chemother 38, 2054-2058 (1994).

55. M. Hafner, M. Niepel, M. Chung, P. K. Sorger, Growth rate inhibition metrics correct for confounders in measuring sensitivity to cancer drugs. Nature Methods 13, 521-527 (2016).

56. P. Yeh, A. I. Tschumi, R. Kishony, Functional classification of drugs by properties of their pairwise interactions. Nature Genetics 38, 489-494 (2006).

57. M. Cokol, H. N. Chua, M. Tasan, B. Mutlu, Z. B. Weinstein, Y. Suzuki, M. E. Nergiz, M. Costanzo, A. Baryshnikova, G. Giaever, C. Nislow, C. L. Myers, B. J. Andrews, C. Boone, F. P. Roth, Systematic exploration of synergistic drug pairs. Mol Syst Biol 7, 544 (2011).

58. S. Chandrasekaran, M. Cokol-Cakmak, N. Sahin, K. Yilancioglu, H. Kazan, J. J. Collins, M. Cokol, Chemogenomics and orthology-based design of antibiotic combination therapies. Molecular systems biology 12, 872-872 (2016).

59. D. J. Mason, I. Stott, S. Ashenden, Z. B. Weinstein, I. Karakoc, S. Meral, N. Kuru, A. Bender, M. Cokol, Prediction of Antibiotic Interactions Using Descriptors Derived from Molecular Structure. J Med Chem 60, 3902-3912 (2017).

60. M. Cokol, C. Li, S. Chandrasekaran, Chemogenomic model identifies synergistic drug combinations robust to the pathogen microenvironment. PLOS Computational Biology 14, e1006677 (2019). 
61. R. Richards, H. R. Schwartz, M. E. Honeywell, M. S. Stewart, P. Cruz-Gordillo, A. J. Joyce, B. D. Landry, M. J. Lee, Drug antagonism and single-agent dominance result from differences in death kinetics. Nat Chem Biol 16, 791-800 (2020).

62. J.-P. Lanoix, R. E. Chaisson, E. L. Nuermberger, Shortening Tuberculosis Treatment With Fluoroquinolones: Lost in Translation? Clinical Infectious Diseases 62, 484-490 (2015).

63. D. A. Mitchison, G. R. Davies, Assessment of the Efficacy of New Anti-Tuberculosis Drugs. Open Infect Dis J 2, 59-76 (2008).

64. R. Tasneen, F. Betoudji, S. Tyagi, S. Y. Li, K. Williams, P. J. Converse, V. Dartois, T. Yang, C. M. Mendel, K. E. Mdluli, E. L. Nuermberger, Contribution of Oxazolidinones to the Efficacy of Novel Regimens Containing Bedaquiline and Pretomanid in a Mouse Model of Tuberculosis. Antimicrob Agents Chemother 60, 270-277 (2016).

65. J. Xu, S. Y. Li, D. V. Almeida, R. Tasneen, K. Barnes-Boyle, P. J. Converse, A. M. Upton, K. Mdluli, N. Fotouhi, E. L. Nuermberger, Contribution of Pretomanid to Novel Regimens Containing Bedaquiline with either Linezolid or Moxifloxacin and Pyrazinamide in Murine Models of Tuberculosis. Antimicrob Agents Chemother 63, (2019).

66. M. A. De Groote, J. C. Gilliland, C. L. Wells, E. J. Brooks, L. K. Woolhiser, V. Gruppo, C. A. Peloquin, I. M. Orme, A. J. Lenaerts, Comparative studies evaluating mouse models used for efficacy testing of experimental drugs against Mycobacterium tuberculosis. Antimicrob Agents Chemother 55, 1237-1247 (2011).

67. E. L. Nuermberger, T. Yoshimatsu, S. Tyagi, R. J. O'Brien, A. N. Vernon, R. E. Chaisson, W. R. Bishai, J. H. Grosset, Moxifloxacin-containing regimen greatly reduces 
time to culture conversion in murine tuberculosis. Am J Respir Crit Care Med 169, 421426 (2004).

68. E. L. Nuermberger, T. Yoshimatsu, S. Tyagi, K. Williams, I. Rosenthal, R. J. O'Brien, A. A. Vernon, R. E. Chaisson, W. R. Bishai, J. H. Grosset, Moxifloxacin-containing regimens of reduced duration produce a stable cure in murine tuberculosis. Am J Respir Crit Care Med 170, 1131-1134 (2004).

69. I. M. Rosenthal, M. Zhang, K. N. Williams, C. A. Peloquin, S. Tyagi, A. A. Vernon, W. R. Bishai, R. E. Chaisson, J. H. Grosset, E. L. Nuermberger, Daily dosing of rifapentine cures tuberculosis in three months or less in the murine model. PLoS Med 4, e344 (2007).

70. M. A. De Groote, V. Gruppo, L. K. Woolhiser, I. M. Orme, J. C. Gilliland, A. J. Lenaerts, Importance of confirming data on the in vivo efficacy of novel antibacterial drug regimens against various strains of Mycobacterium tuberculosis. Antimicrob Agents Chemother 56, 731-738 (2012).

71. B. C. Mourik, G. J. de Knegt, A. Verbon, J. W. Mouton, H. I. Bax, J. E. M. de Steenwinkel, Assessment of Bactericidal Drug Activity and Treatment Outcome in a Mouse Tuberculosis Model Using a Clinical Beijing Strain. Antimicrob Agents Chemother 61, (2017).

72. C. T. Meyer, D. J. Wooten, B. B. Paudel, J. Bauer, K. N. Hardeman, D. Westover, C. M. Lovly, L. A. Harris, D. R. Tyson, V. Quaranta, Quantifying Drug Combination Synergy along Potency and Efficacy Axes. Cell Syst 8, 97-108 e116 (2019).

73. A. C. Palmer, C. Chidley, P. K. Sorger, A curative combination cancer therapy achieves high fractional cell killing through low cross-resistance and drug additivity. eLife $\mathbf{8}$, e50036 (2019) 
74. P. Sen, A. Saha, N. M. Dixit, You Cannot Have Your Synergy and Efficacy Too. Trends Pharmacol Sci 40, 811-817 (2019).

75. J. Harper, C. Skerry, S. L. Davis, R. Tasneen, M. Weir, I. Kramnik, W. R. Bishai, M. G. Pomper, E. L. Nuermberger, S. K. Jain, Mouse model of necrotic tuberculosis granulomas develops hypoxic lesions. J Infect Dis 205, 595-602 (2012).

76. S. M. Irwin, E. Driver, E. Lyon, C. Schrupp, G. Ryan, M. Gonzalez-Juarrero, R. J. Basaraba, E. L. Nuermberger, A. J. Lenaerts, Presence of multiple lesion types with vastly different microenvironments in $\mathrm{C} 3 \mathrm{HeB} / \mathrm{FeJ}$ mice following aerosol infection with $<\mathrm{em}>$ Mycobacterium tuberculosis</em>. Disease Models \&amp; Mechanisms 8, 591$602(2015)$.

77. S. M. Irwin, B. Prideaux, E. R. Lyon, M. D. Zimmerman, E. J. Brooks, C. A. Schrupp, C. Chen, M. J. Reichlen, B. C. Asay, M. I. Voskuil, E. L. Nuermberger, K. Andries, M. A. Lyons, V. Dartois, A. J. Lenaerts, Bedaquiline and Pyrazinamide Treatment Responses Are Affected by Pulmonary Lesion Heterogeneity in Mycobacterium tuberculosis Infected C3HeB/FeJ Mice. ACS Infect Dis 2, 251-267 (2016).

78. V. Dartois, The path of anti-tuberculosis drugs: from blood to lesions to mycobacterial cells. Nat Rev Microbiol 12, 159-167 (2014).

79. A. C. Palmer, P. K. Sorger, Combination Cancer Therapy Can Confer Benefit via Patientto-Patient Variability without Drug Additivity or Synergy. Cell 171, 1678-1691.e1613 (2017).

80. K. M. Bigelow, R. Tasneen, Y. S. Chang, K. E. Dooley, E. L. Nuermberger, Preserved Efficacy and Reduced Toxicity with Intermittent Linezolid Dosing in Combination with 
Bedaquiline and Pretomanid in a Murine Tuberculosis Model. Antimicrobial Agents and Chemotherapy 64, e01178-01120 (2020).

81. K. M. Wilburn, R. A. Fieweger, B. C. VanderVen, Cholesterol and fatty acids grease the wheels of Mycobacterium tuberculosis pathogenesis. Pathog Dis 76, (2018).

82. G. Gago, L. Diacovich, H. Gramajo, Lipid metabolism and its implication in mycobacteria-host interaction. Curr Opin Microbiol 41, 36-42 (2018).

83. J. E. Griffin, A. K. Pandey, S. A. Gilmore, V. Mizrahi, J. D. McKinney, C. R. Bertozzi, C. M. Sassetti, Cholesterol catabolism by Mycobacterium tuberculosis requires transcriptional and metabolic adaptations. Chem Biol 19, 218-227 (2012).

84. D. G. Russell, B. C. VanderVen, W. Lee, R. B. Abramovitch, M. J. Kim, S. Homolka, S. Niemann, K. H. Rohde, Mycobacterium tuberculosis wears what it eats. Cell Host Microbe 8, 68-76 (2010).

85. A. K. Pandey, C. M. Sassetti, Mycobacterial persistence requires the utilization of host cholesterol. Proc Natl Acad Sci U S A 105, 4376-4380 (2008).

86. J. C. Chang, M. D. Miner, A. K. Pandey, W. P. Gill, N. S. Harik, C. M. Sassetti, D. R. Sherman, igr Genes and Mycobacterium tuberculosis cholesterol metabolism. J Bacteriol 191, 5232-5239 (2009).

87. R. R. Lovewell, C. M. Sassetti, B. C. VanderVen, Chewing the fat: lipid metabolism and homeostasis during M. tuberculosis infection. Curr Opin Microbiol 29, 30-36 (2016).

88. S. Puckett, C. Trujillo, H. Eoh, J. Marrero, J. Spencer, M. Jackson, D. Schnappinger, K. Rhee, S. Ehrt, Inactivation of fructose-1,6-bisphosphate aldolase prevents optimal cocatabolism of glycolytic and gluconeogenic carbon substrates in Mycobacterium tuberculosis. PLoS Pathog 10, e1004144 (2014). 
89. T. Beites, K. O'Brien, D. Tiwari, C. A. Engelhart, S. Walters, J. Andrews, H. J. Yang, M.

L. Sutphen, D. M. Weiner, E. K. Dayao, M. Zimmerman, B. Prideaux, P. V. Desai, T.

Masquelin, L. E. Via, V. Dartois, H. I. Boshoff, C. E. Barry, 3rd, S. Ehrt, D.

Schnappinger, Plasticity of the Mycobacterium tuberculosis respiratory chain and its

impact on tuberculosis drug development. Nat Commun 10, 4970 (2019).

90. S. Ehrt, D. Schnappinger, Mycobacterium tuberculosis virulence: lipids inside and out.

Nat Med 13, 284-285 (2007).

91. J. P. Sarathy, V. Dartois, Caseum: a Niche for Mycobacterium tuberculosis Drug-

Tolerant Persisters. Clin Microbiol Rev 33, (2020).

92. M. Pai, M. A. Behr, D. Dowdy, K. Dheda, M. Divangahi, C. C. Boehme, A. Ginsberg, S.

Swaminathan, M. Spigelman, H. Getahun, D. Menzies, M. Raviglione, Tuberculosis.

Nature Reviews Disease Primers 2, 16076 (2016).

93. J. P. Sarathy, L. E. Via, D. Weiner, L. Blanc, H. Boshoff, E. A. Eugenin, C. E. Barry, 3rd, V. A. Dartois, Extreme Drug Tolerance of Mycobacterium tuberculosis in Caseum. Antimicrob Agents Chemother 62, (2018).

94. Y. Liu, S. Tan, L. Huang, R. B. Abramovitch, K. H. Rohde, M. D. Zimmerman, C. Chen, V. Dartois, B. C. VanderVen, D. G. Russell, Immune activation of the host cell induces drug tolerance in Mycobacterium tuberculosis both in vitro and in vivo. J Exp Med 213, 809-825 (2016).

95. S. H. Gillespie, A. M. Crook, T. D. McHugh, C. M. Mendel, S. K. Meredith, S. R. Murray, F. Pappas, P. P. Phillips, A. J. Nunn, R. E. Consortium, Four-month moxifloxacin-based regimens for drug-sensitive tuberculosis. N Engl J Med 371, 15771587 (2014). 
96. Y. L. Xie, V. R. de Jager, R. Y. Chen, L. E. Dodd, P. Paripati, L. E. Via, D. Follmann, J. Wang, K. Lumbard, S. Lahouar, S. T. Malherbe, J. Andrews, X. Yu, L. C. Goldfeder, Y. Cai, K. Arora, A. G. Loxton, N. Vanker, M. Duvenhage, J. Winter, T. Song, G. Walzl, A. H. Diacon, C. E. Barry, Fourteen-day PET/CT imaging to monitor drug combination activity in treated individuals with tuberculosis. Science Translational Medicine 13, eabd7618 (2021).

97. clinicaltrials.gov. National Library of Medicine. (2020). NCT03338621. Trial to Evaluate the Efficacy, Safety and Tolerability of BPaMZ in Drug-Sensitive (DS-TB) Adult Patients and Drug-Resistant (DR-TB) Adult Patients. https://ClinicalTrials.gov/show/NCT03338621

98. K. E. Dooley, D. Hanna, V. Mave, K. Eisenach, R. M. Savic, Advancing the development of new tuberculosis treatment regimens: The essential role of translational and clinical pharmacology and microbiology. PLoS Med 16, e1002842 (2019).

99. I. H. Bartelink, N. Zhang, R. J. Keizer, N. Strydom, P. J. Converse, K. E. Dooley, E. L. Nuermberger, R. M. Savic, New Paradigm for Translational Modeling to Predict Longterm Tuberculosis Treatment Response. Clin Transl Sci 10, 366-379 (2017).

100. J. B. Michel, P. J. Yeh, R. Chait, R. C. Moellering, Jr., R. Kishony, Drug interactions modulate the potential for evolution of resistance. Proc Natl Acad Sci US A 105, 1491814923 (2008).

101. A. R. M. Coates, Y. Hu, J. Holt, P. Yeh, Antibiotic combination therapy against resistant bacterial infections: synergy, rejuvenation and resistance reduction. Expert Rev Anti Infect Ther 18, 5-15 (2020). 
102. J. Liu, O. Gefen, I. Ronin, M. Bar-Meir, N. Q. Balaban, Effect of tolerance on the evolution of antibiotic resistance under drug combinations. Science 367, 200-204 (2020).

103. B. K. Greenfield, S. Shaked, C. F. Marrs, P. Nelson, I. Raxter, C. Xi, T. E. McKone, O. Jolliet, Modeling the Emergence of Antibiotic Resistance in the Environment: an Analytical Solution for the Minimum Selection Concentration. Antimicrob Agents Chemother 62, (2018).

104. S. Ma, S. Jaipalli, J. Larkins-Ford, J. Lohmiller, B. B. Aldridge, D. R. Sherman, S. Chandrasekaran, Transcriptomic Signatures Predict Regulators of Drug Synergy and Clinical Regimen Efficacy against Tuberculosis. mBio 10, (2019).

105. N. Andreu, A. Zelmer, T. Fletcher, P. T. Elkington, T. H. Ward, J. Ripoll, T. Parish, G. J. Bancroft, U. Schaible, B. D. Robertson, S. Wiles, Optimisation of bioluminescent reporters for use with mycobacteria. PLoS One 5, e10777 (2010).

106. S. A. Stanley, S. S. Grant, T. Kawate, N. Iwase, M. Shimizu, C. Wivagg, M. Silvis, E. Kazyanskaya, J. Aquadro, A. Golas, M. Fitzgerald, H. Dai, L. Zhang, D. T. Hung, Identification of novel inhibitors of M. tuberculosis growth using whole cell based highthroughput screening. ACS Chem Biol 7, 1377-1384 (2012).

107. S. Sharma, E. Gelman, C. Narayan, D. Bhattacharjee, V. Achar, V. Humnabadkar, V. Balasubramanian, V. Ramachandran, N. Dhar, N. Dinesh, Simple and rapid method to determine antimycobacterial potency of compounds by using autoluminescent Mycobacterium tuberculosis. Antimicrob Agents Chemother 58, 5801-5808 (2014).

108. T. Zhang, S.-Y. Li, E. L. Nuermberger, Autoluminescent Mycobacterium tuberculosis for Rapid, Real-Time, Non-Invasive Assessment of Drug and Vaccine Efficacy. PLOS ONE 7, e29774 (2012). 
109. D. Russ, R. Kishony, Additivity of inhibitory effects in multidrug combinations. Nat Microbiol 3, 1339-1345 (2018).

110. S. Dray, J. Josse, Principal component analysis with missing values: a comparative survey of methods. Plant Ecology 216, 657-667 (2014).

111. J. Foucquier, M. Guedj, Analysis of drug combinations: current methodological landscape. Pharmacol Res Perspect 3, e00149 (2015).

112. S. Y. Li, R. Tasneen, S. Tyagi, H. Soni, P. J. Converse, K. Mdluli, E. L. Nuermberger, Bactericidal and Sterilizing Activity of a Novel Regimen with Bedaquiline, Pretomanid, Moxifloxacin, and Pyrazinamide in a Murine Model of Tuberculosis. Antimicrob Agents Chemother 61, (2017).

113. R. Tasneen, K. Williams, O. Amoabeng, A. Minkowski, K. E. Mdluli, A. M. Upton, E. L. Nuermberger, Contribution of the nitroimidazoles PA-824 and TBA-354 to the activity of novel regimens in murine models of tuberculosis. Antimicrob Agents Chemother 59, 129135 (2015).

114. K. Williams, A. Minkowski, O. Amoabeng, C. A. Peloquin, D. Taylor, K. Andries, R. S. Wallis, K. E. Mdluli, E. L. Nuermberger, Sterilizing activities of novel combinations lacking first- and second-line drugs in a murine model of tuberculosis. Antimicrob Agents Chemother 56, 3114-3120 (2012).

115. K. Andries, T. Gevers, N. Lounis, Bactericidal potencies of new regimens are not predictive of their sterilizing potencies in a murine model of tuberculosis. Antimicrob Agents Chemother 54, 4540-4544 (2010).

116. J. P. Lanoix, R. Tasneen, P. O'Brien, J. Sarathy, H. Safi, M. Pinn, D. Alland, V. Dartois, E. Nuermberger, High Systemic Exposure of Pyrazinoic Acid Has Limited 
Antituberculosis Activity in Murine and Rabbit Models of Tuberculosis. Antimicrob Agents Chemother 60, 4197-4205 (2016).

117. E. Nuermberger, I. Rosenthal, S. Tyagi, K. N. Williams, D. Almeida, C. A. Peloquin, W. R. Bishai, J. H. Grosset, Combination chemotherapy with the nitroimidazopyran PA-824 and first-line drugs in a murine model of tuberculosis. Antimicrob Agents Chemother 50, 2621-2625 (2006).

118. I. M. Rosenthal, R. Tasneen, C. A. Peloquin, M. Zhang, D. Almeida, K. E. Mdluli, P. C. Karakousis, J. H. Grosset, E. L. Nuermberger, Dose-ranging comparison of rifampin and rifapentine in two pathologically distinct murine models of tuberculosis. Antimicrob Agents Chemother 56, 4331-4340 (2012).

119. V. Saini, N. C. Ammerman, Y. S. Chang, R. Tasneen, R. E. Chaisson, S. Jain, E. Nuermberger, J. H. Grosset, Treatment-Shortening Effect of a Novel Regimen Combining Clofazimine and High-Dose Rifapentine in Pathologically Distinct Mouse Models of Tuberculosis. Antimicrob Agents Chemother 63, (2019).

120. B. Y. Lee, D. L. Clemens, A. Silva, B. J. Dillon, S. Maslesa-Galic, S. Nava, C. M. Ho, M. A. Horwitz, Ultra-rapid near universal TB drug regimen identified via parabolic response surface platform cures mice of both conventional and high susceptibility. PLoS One 13, e0207469 (2018).

121. I. M. Rosenthal, M. Zhang, D. Almeida, J. H. Grosset, E. L. Nuermberger, Isoniazid or moxifloxacin in rifapentine-based regimens for experimental tuberculosis? Am J Respir Crit Care Med 178, 989-993 (2008). 
122. B. Y. Lee, D. L. Clemens, A. Silva, B. J. Dillon, S. Maslesa-Galic, S. Nava, X. Ding, C.

M. Ho, M. A. Horwitz, Drug regimens identified and optimized by output-driven platform markedly reduce tuberculosis treatment time. Nat Commun 8, 14183 (2017).

123. N. C. Ammerman, R. V. Swanson, E. M. Bautista, D. V. Almeida, V. Saini, T. F.

Omansen, H. Guo, Y. S. Chang, S. Y. Li, A. Tapley, R. Tasneen, S. Tyagi, F. Betoudji, C. Moodley, B. Ngcobo, L. Pillay, L. A. Bester, S. D. Singh, R. E. Chaisson, E. Nuermberger, J. H. Grosset, Impact of Clofazimine Dosing on Treatment Shortening of the First-Line Regimen in a Mouse Model of Tuberculosis. Antimicrob Agents Chemother 62, (2018).

124. S. Tyagi, N. C. Ammerman, S. Y. Li, J. Adamson, P. J. Converse, R. V. Swanson, D. V. Almeida, J. H. Grosset, Clofazimine shortens the duration of the first-line treatment regimen for experimental chemotherapy of tuberculosis. Proc Natl Acad Sci US A 112, 869-874 (2015).

125. S. Y. Li, S. M. Irwin, P. J. Converse, K. E. Mdluli, A. J. Lenaerts, E. L. Nuermberger, Evaluation of moxifloxacin-containing regimens in pathologically distinct murine tuberculosis models. Antimicrob Agents Chemother 59, 4026-4030 (2015).

126. K. N. Williams, S. J. Brickner, C. K. Stover, T. Zhu, A. Ogden, R. Tasneen, S. Tyagi, J. H. Grosset, E. L. Nuermberger, Addition of PNU-100480 to first-line drugs shortens the time needed to cure murine tuberculosis. Am J Respir Crit Care Med 180, 371-376 (2009).

127. B. C. Mourik, R. J. Svensson, G. J. de Knegt, H. I. Bax, A. Verbon, U. S. H. Simonsson, J. E. M. de Steenwinkel, Improving treatment outcome assessment in a mouse tuberculosis model. Sci Rep 8, 5714 (2018). 
128. D. L. Clemens, B. Y. Lee, A. Silva, B. J. Dillon, S. Maslesa-Galic, S. Nava, X. Ding, C.

M. Ho, M. A. Horwitz, Artificial intelligence enabled parabolic response surface platform identifies ultra-rapid near-universal TB drug treatment regimens comprising approved drugs. PLoS One 14, e0215607 (2019).

129. G. T. Robertson, M. S. Scherman, D. F. Bruhn, J. Liu, C. Hastings, M. R. McNeil, M. M. Butler, T. L. Bowlin, R. B. Lee, R. E. Lee, A. J. Lenaerts, Spectinamides are effective partner agents for the treatment of tuberculosis in multiple mouse infection models. $J$ Antimicrob Chemother 72, 770-777 (2017).

130. K. N. Williams, C. K. Stover, T. Zhu, R. Tasneen, S. Tyagi, J. H. Grosset, E. Nuermberger, Promising antituberculosis activity of the oxazolidinone PNU-100480 relative to that of linezolid in a murine model. Antimicrob Agents Chemother 53, 13141319 (2009).

131. J. P. Lanoix, F. Betoudji, E. Nuermberger, Sterilizing Activity of Pyrazinamide in Combination with First-Line Drugs in a C3HeB/FeJ Mouse Model of Tuberculosis. Antimicrob Agents Chemother 60, 1091-1096 (2016).

132. L. Fattorini, D. Tan, E. Iona, M. Mattei, F. Giannoni, L. Brunori, S. Recchia, G. Orefici, Activities of moxifloxacin alone and in combination with other antimicrobial agents against multidrug-resistant Mycobacterium tuberculosis infection in BALB/c mice. Antimicrob Agents Chemother 47, 360-362 (2003).

133. M. Cynamon, M. R. Sklaney, C. Shoen, Gatifloxacin in combination with rifampicin in a murine tuberculosis model. J Antimicrob Chemother 60, 429-432 (2007). 
134. B. V. Nikonenko, M. Protopopova, R. Samala, L. Einck, C. A. Nacy, Drug therapy of experimental tuberculosis (TB): improved outcome by combining SQ109, a new diamine antibiotic, with existing TB drugs. Antimicrob Agents Chemother 51, 1563-1565 (2007).

Acknowledgments: We thank members of the Aldridge laboratory, V. Dartois, R. Isberg, K. Mdluli, J. Mecsas, A. Palmer, J. Silverman, and S. Tan for insightful discussion. Bedaquiline and pretomanid were provided by the NIH AIDS Reagent Program and the TB Alliance, respectively. pMV306hsp+LuxG13 was a gift from Brian Robertson and Siouxsie Wiles. Funding: This work is funded by BMGF OPP1189457 to BA and NIH grant 1U54CA225088: Systems Pharmacology of Therapeutic and Adverse Responses to Immune Checkpoint and Small Molecule Drugs for AS. Author contributions: JLF, TG, NV, YD, and BBA conceived and designed the experiments. JLF, TG, NV, and YD performed the experiments. JLF, TG, NV, YD, MO, AS, and BBA conceived and designed the computational analysis. JLF, TG, NV, YD, and MO performed the computational analysis. The manuscript was written by JLF and BBA. All authors contributed to interpretation of the results and editing of the manuscript. Competing interests: All authors declare that they have no competing interests. 
Figures:

Fig. 1.
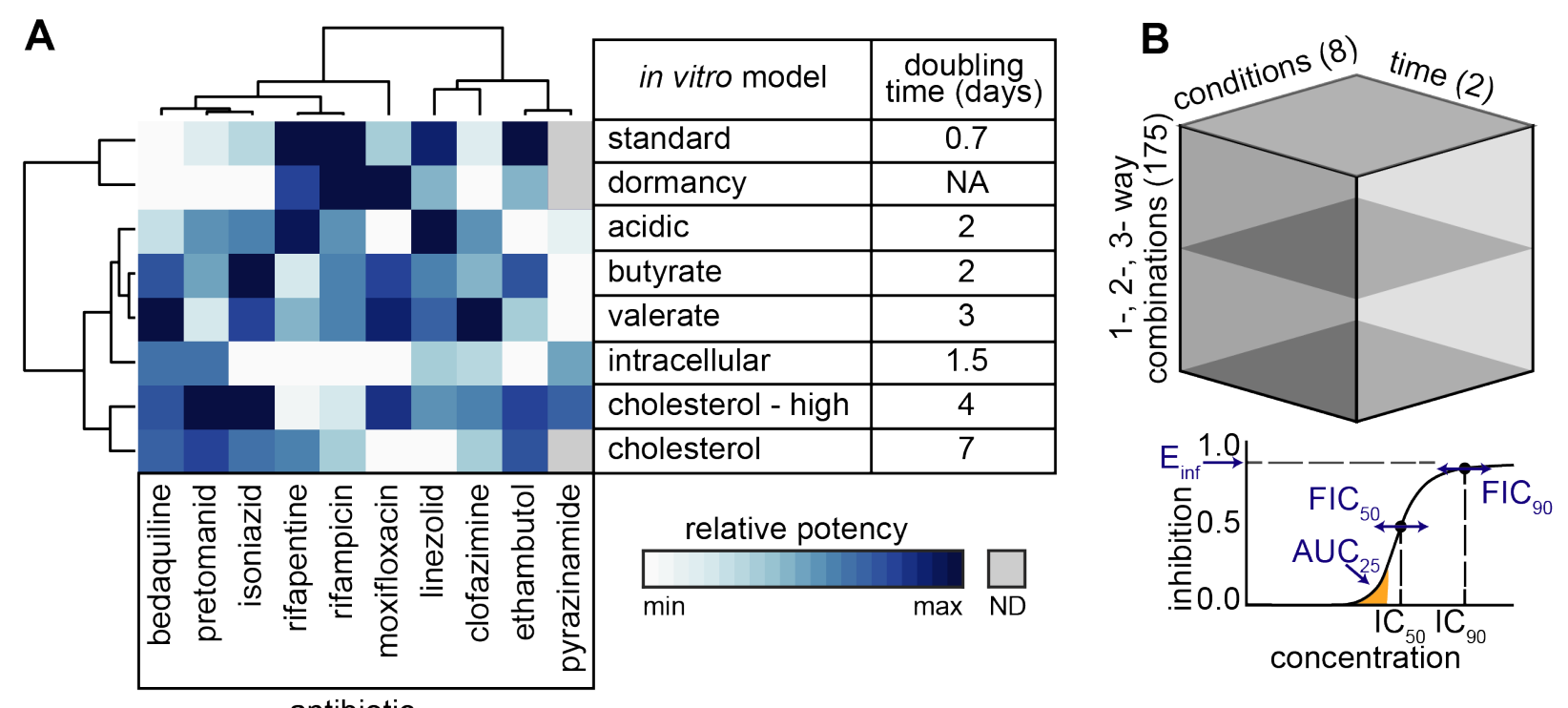

antibiotic

Fig. 1. 10-drug DiaMOND compendium of Mtb response to drug combination treatment. (A)

Relative potencies of the ten compendium drugs in eight in vitro conditions (IC90, terminal time point; left) with doubling times for each condition in untreated Mtb (right).

Hierarchical clustering of potencies as calculated with cosine distances and average linkage. $\mathrm{IC}_{90}$ is color scaled (log10 transformation) within each drug (Table S3). ND =

Not determined. NA = not applicable. (B) Metrics from DiaMOND dose response curves.

$\mathrm{IC}_{50}$ and $\mathrm{IC}_{90}$ are used to calculate drug interactions at the $50 \%$ and $90 \%$ levels of growth inhibition ( FIC $_{50}$ and FIC $_{90}$, respectively). Three potency metrics are derived: $\mathrm{AUC}_{25}=$ normalized area under the curve until $25 \%$ inhibition, $\mathrm{E}_{\text {inf }}=$ theoretical maximum inhibition, and $\mathrm{GR}_{\mathrm{inf}}=$ theoretical maximum normalized growth rate inhibition (Box and Materials and Methods). (C) Schematic data cube of the DiaMOND compendium. Mtb were treated with all 1-, 2-, and 3-way drug combinations (175 combinations) among 10- 
drugs in dose responses measured in 10-dose resolution in at least biological duplicate.

Dose response measurements were made in eight in vitro models and at 3-4 time points, but we focus on 1-2 time points for analysis; therefore, this data cube represents $\sim 25 \%$ of the total measurements made. 
Fig. 2.

A

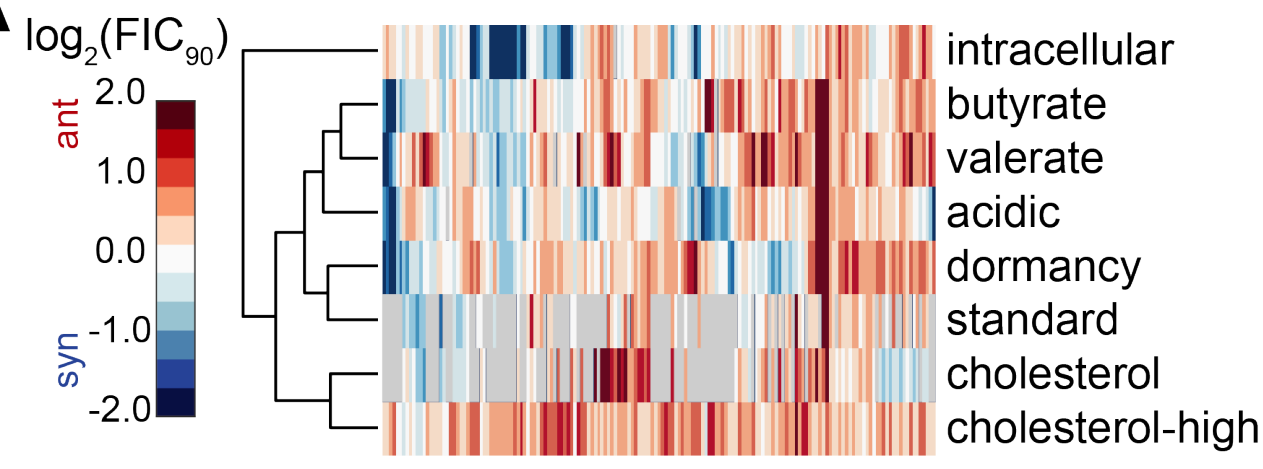

C

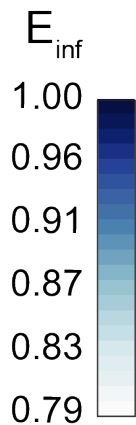

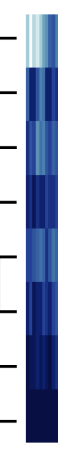

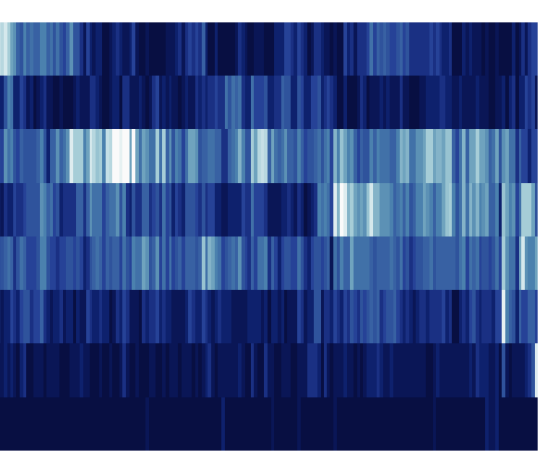

intracellular

acidic

cholesterol

standard

valerate

butyrate

dormancy

cholesterol-high

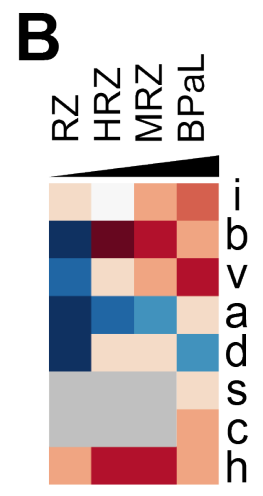

D
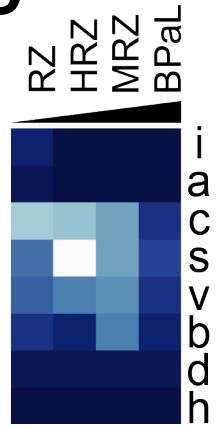

Fig. 2. Drug interaction and potency patterns in the DiaMOND compendium. (A) Drug interaction profiles of all 2- and 3-drug combinations among the ten compendium drugs across the in vitro models $\left(\log _{2}\left(\mathrm{FIC}_{90}\right)\right.$ at the terminal time point, clustered based on cosine distance). (B) Drug interaction profiles of selected drug combinations ordered by mouse relapse outcome efficacy $(22,64-71)$. See Table 1 for drug combination abbreviations. (C) Drug combination potency profiles of all 2- and 3-drug combinations among the ten compendium drugs across the in vitro models ( $\mathrm{E}_{\text {inf }}$ at the terminal time point, clustered based on cosine distance). (D) Drug interaction profiles of selected drug 
bioRxiv preprint doi: https://doi.org/10.1101/2021.02.03.429579; this version posted February 6, 2021. The copyright holder for this preprint (which was not certified by peer review) is the author/funder, who has granted bioRxiv a license to display the preprint in perpetuity. It is made available under aCC-BY-NC-ND 4.0 International license.

combinations ordered by mouse relapse outcome efficacy $(22,64-71)$. See Table 1 for drug combination abbreviations. gray $=$ ND. 
Fig. 3.
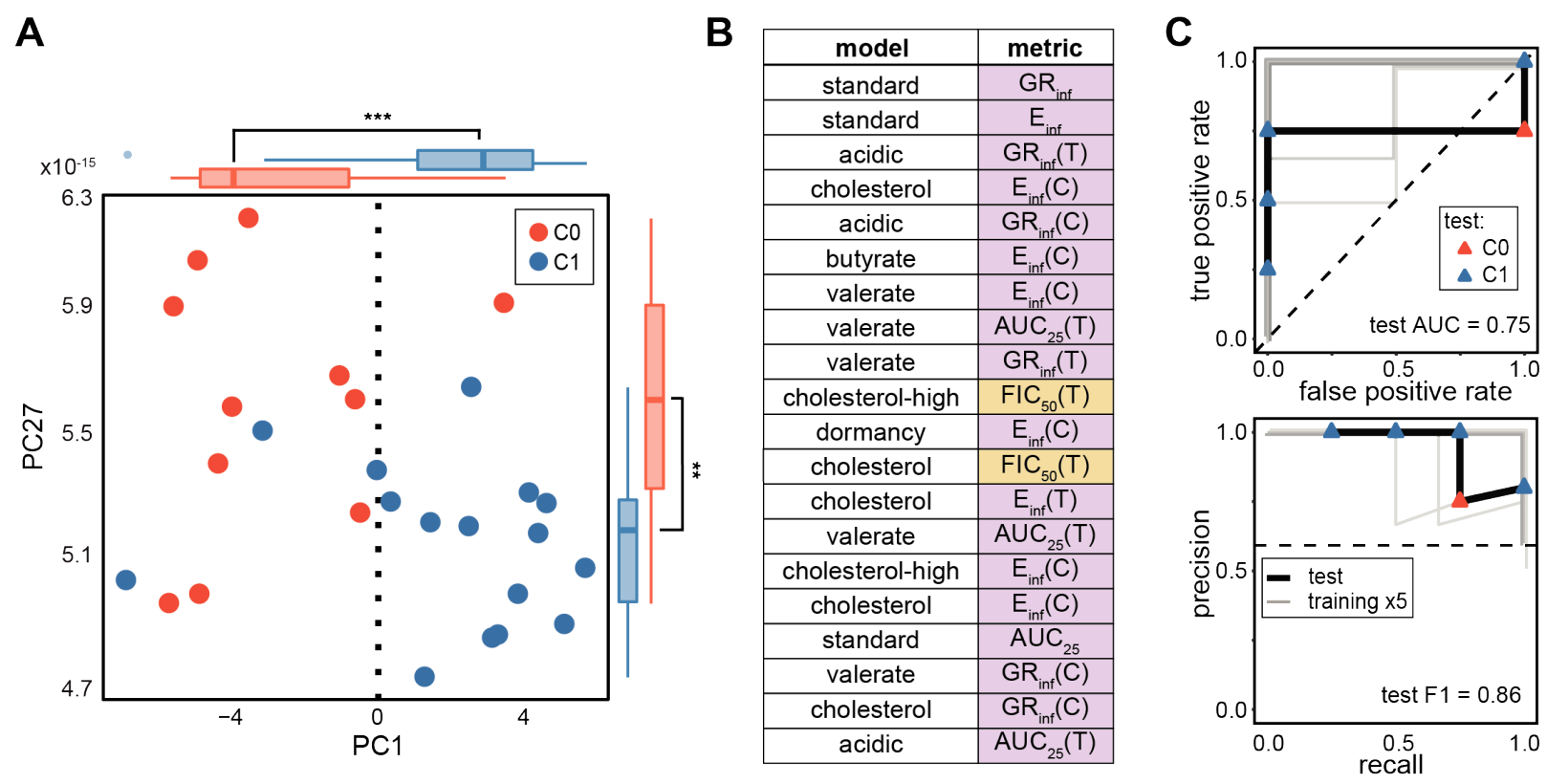

Fig. 3. Prediction of combination treatment outcomes in the RMM with DiaMOND data. (A) PCA of DiaMOND data labeled by outcome in the RMM $(\mathrm{C} 1$ is better than the standard of care, blue; $\mathrm{C} 0$ is standard of care or worse, red) with the most discriminating two PCs shown. Outside the scatter plot are box and whisker plots of the distributions of $\mathrm{C} 1$ and C0 combinations along PC1 and PC27 (Wilcoxon rank-sum test: *** p<0.005. ** $\mathrm{p}<0.01$ ). (B) Highest weighted features in $\mathrm{PC1}$ with in vitro model (abbreviations in Fig. 1A) and metric type indicated. Metrics are classified and shaded according to whether they are related to drug combination potency (purple: $\mathrm{AUC}_{25}, \mathrm{E}_{\mathrm{inf}}$, and $\mathrm{GR}_{\text {inf }}$ ) or drug interaction (orange: FIC $_{50}$ and $\mathrm{FIC}_{90}$ ). (C) ROC curves (top panel, Table 1) and PR curves (bottom panel, Table 1) of a random forest-based classifier trained on all eight conditions in the DiaMOND compendium. The model is tested with high-order combinations (4- and 5-drug combinations) that were excluded from training. Training (gray lines each show one of five cross validations; lines are slightly offset to aid 
visualization) and test (black) performances are shown with lines. Test combinations are colored by outcome class as in (A). Performance metrics are shown on plots for test data (Area Under the ROC curve (AUC) and F1, harmonic mean of precision and recall, Table 1). Dashed lines indicate theoretical "no-skill" model performance. 
Fig. 4.

A
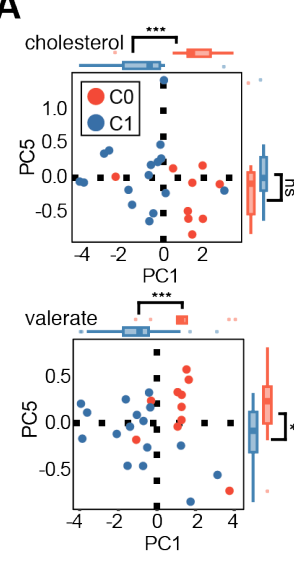
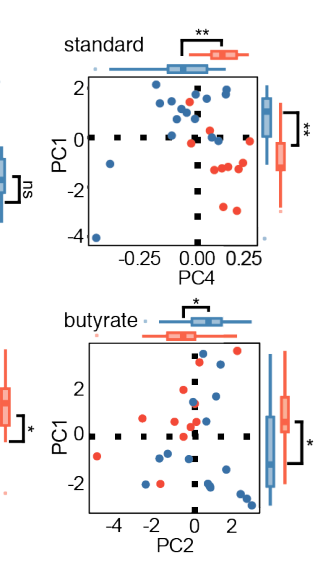
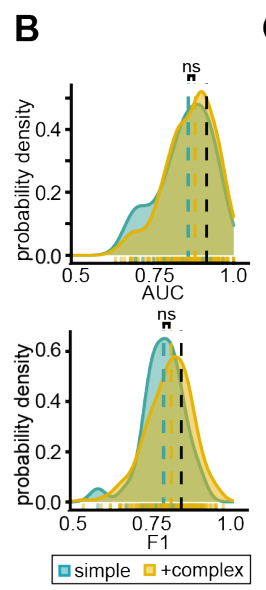

C acidic + cholesterol
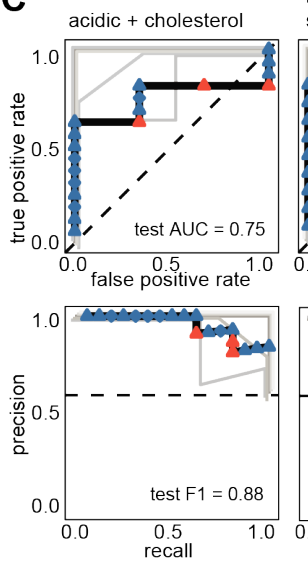

butyrate + cholesterol + acidic + cholesterol + standard + valerate standard + valerate
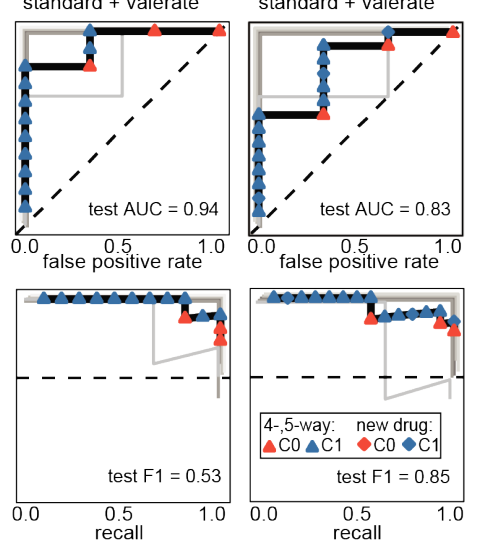

Fig. 4. Prediction of combination therapy outcomes in the RMM using fewer in vitro models. (A) PCA plot of DiaMOND data labeled by outcome in the RMM (plots are labeled as in Fig. 3A). Each subplot is DiaMOND data from one in vitro condition plotted in the PC space with the most discriminating two PCs shown for each model. Outside the scatter plot are box and whisker plots of the distributions of $\mathrm{C} 1$ and $\mathrm{C} 0$ combinations along $\mathrm{PC} 1$ and $\mathrm{PC} 2$ (Wilcoxon rank test: $\left.* * * \mathrm{p}<0.005 .{ }^{* *} \mathrm{p}<0.01 .{ }^{*} \mathrm{p}<0.05 . \mathrm{ns} \mathrm{p}>0.05\right)$. (B) Density distribution plots of estimated classifier performances from systematic survey of all possible in vitro model subsets. Distributions of ROC AUC (top) and F1 (bottom) are separated based on whether technically complex models (intracellular, cholesterol-high, dormancy) are included (yellow) or whether only simple conditions (acidic, butyrate, cholesterol, standard, valerate) are considered. Colored dashed lines indicate mean value for distribution. The estimated performances when using all in vitro models (as in Fig. 3) is shown with black dashed lines. Distributions are compared with a Wilcoxon rank sum test (ns = not significant). (C) Comparison of classification performances of three highperformance random forest classifiers using subsets of simple in vitro models. Training 
(gray lines each show one of five cross validations; lines are slightly offset when they are on top of each other) and test (black) performance is demonstrated with ROC (top) and PR (bottom) curves. Test combinations are colored by outcome class as in panel (A). Plot shapes indicate whether a test combination contained higher-order 4- and 5- drug combinations (triangle) or a combination containing a new drug (diamond-shape) not included in the compendium described in Fig. 1. Dashed lines indicate theoretical "noskill" model performance. 
Fig. 5.

A

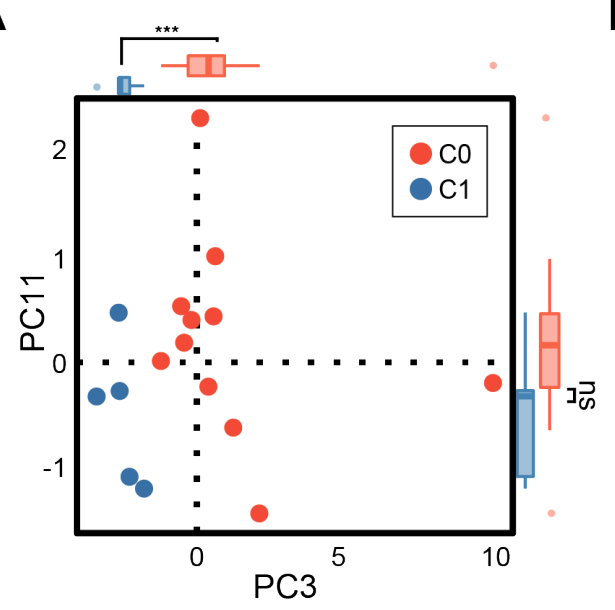

B

\begin{tabular}{|c|c|}
\hline model & metric \\
\hline valerate & $\mathrm{FIC}_{50}(\mathrm{~T})$ \\
\hline butyrate & $\mathrm{FIC}_{50}(\mathrm{~T})$ \\
\hline valerate & $\mathrm{FIC}_{50}(\mathrm{C})$ \\
\hline butyrate & $\mathrm{FIC}_{50}(\mathrm{C})$ \\
\hline acidic & $\mathrm{FIC}_{50}(\mathrm{~T})$ \\
\hline dormancy & $\mathrm{FIC}_{50}(\mathrm{~T})$ \\
\hline dormancy & $\mathrm{FIC}_{90}(\mathrm{~T})$ \\
\hline dormancy & $\mathrm{FIC}_{50}(\mathrm{C})$ \\
\hline acidic & $\mathrm{FIC}_{50}(\mathrm{C})$ \\
\hline butyrate & $\mathrm{FIC}_{90}(\mathrm{~T})$ \\
\hline
\end{tabular}
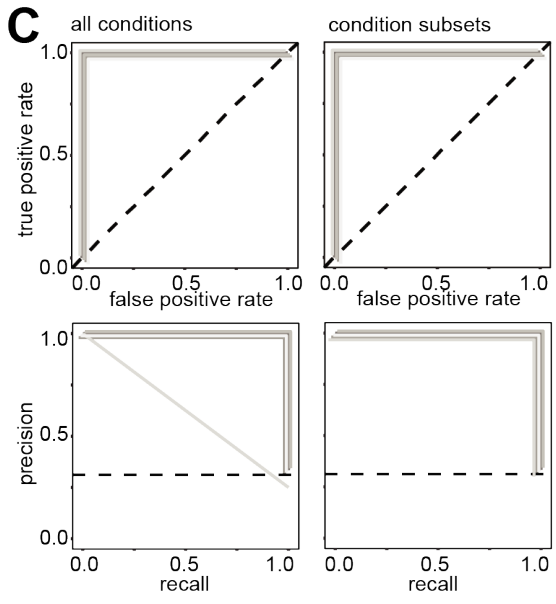

Fig. 5. Signatures of DiaMOND data to describe outcome in the $\mathrm{C} 3 \mathrm{HeB} / \mathrm{FeJ}(\mathrm{BHeB})$ mouse model. (A) PCA plot of DiaMOND data labeled by outcome in the BHeB (plot labels are as in Fig. 3A). (B) Highest weighted features in PC3 with in vitro model and metric type indicated (features are as described in Fig. 3B). (C) Machine learning performance plots for training with 5-fold cross validation (each in a gray line) with ROC (top) and PR (bottom) curves for models trained using all eight conditions (left) and three highperforming subsets of conditions (right: acidic + butyrate + valerate, cholesterol + valerate, and standard + valerate). Subsets had perfect training performance (AUC = 1.0, Table S7). Dashed lines indicate theoretical "no-skill" model performance. 
Fig. 6.
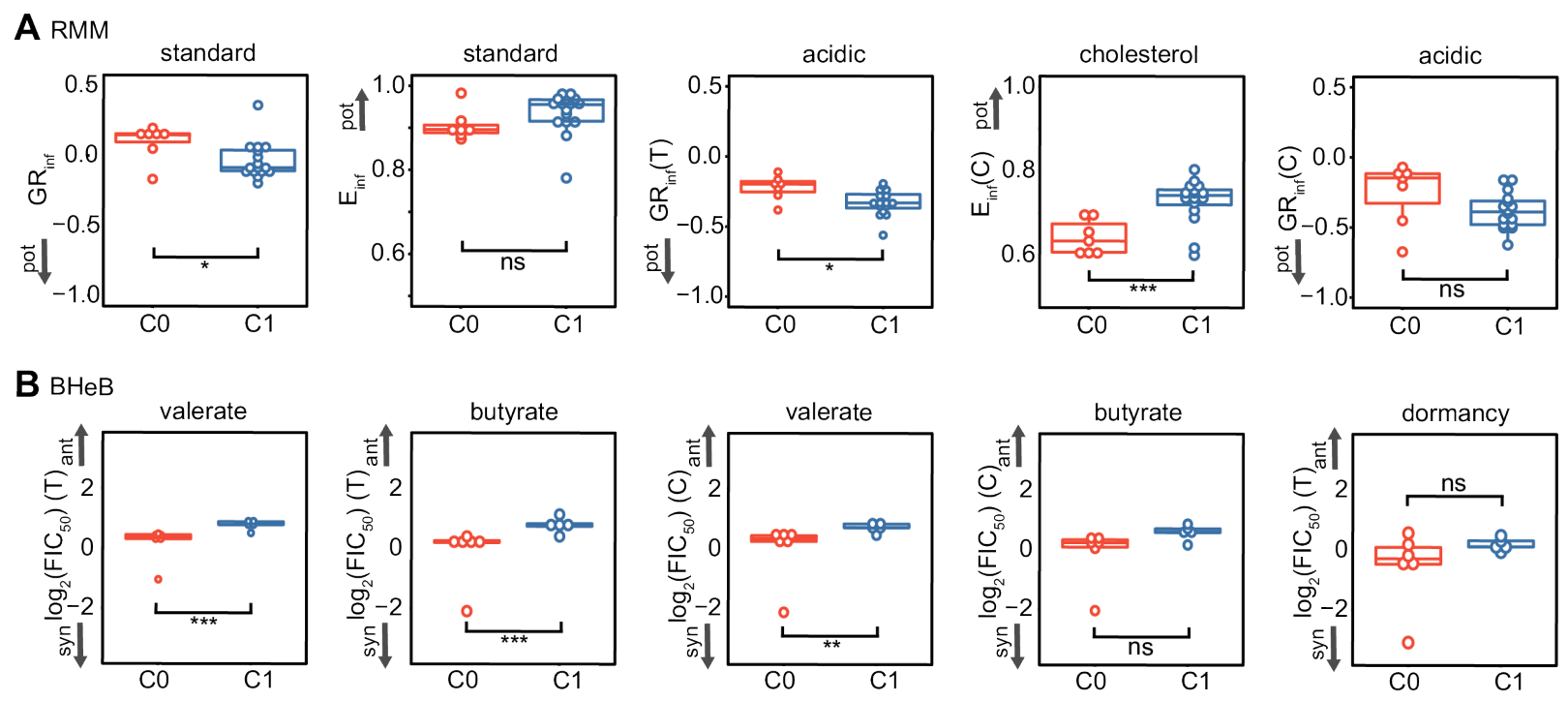

Fig. 6. Properties of signature potency and drug interaction characteristics that describe RMM and $\mathrm{BHeB}$ combination treatment outcomes. Values of the five highest weighted features in the most discriminatory $\mathrm{PC}$ are compared for $\mathrm{C} 1$ and $\mathrm{C} 0$ combinations in the $\mathrm{RMM}$ (A) and BHeB models (B) using dot and box plots. The top features in RMM are potency metrics whereas the top features are drug interaction metrics in BHeB. High vs. low potency (pot) and synergy (syn) vs. antagonism (ant) is indicated with arrows on each subplot. (Wilcoxon rank test: ${ }^{* * *} \mathrm{p}<0.005 .{ }^{* *} \mathrm{p}<0.01 .{ }^{*} \mathrm{p}<0.05 . \mathrm{ns} \mathrm{p}>0.05$ ). 
bioRxiv preprint doi: https://doi.org/10.1101/2021.02.03.429579; this version posted February 6, 2021. The copyright holder for this preprint (which was not certified by peer review) is the author/funder, who has granted bioRxiv a license to display the preprint in perpetuity. It is made available under aCC-BY-NC-ND 4.0 International license.

Box

DiaMOND (Diagonal measurement of $n$-way drug interactions ) is a quantitative framework to efficiently measure drug interactions. The method is based on geometric sampling of traditional combination checkerboards and can be applied to any number of drugs in combination. Optical density $\left(\mathrm{OD}_{600}\right)$ or luminescence measures are normalized to untreated controls and subtracted from 1 to obtain fractional growth inhibition. The concentrations to achieve a particular effect (e.g., concentration to achieve $90 \%$ growth inhibition, $\mathrm{IC}_{\mathrm{o}}$, depicted in blue and orange circles) are experimentally determined for all single drugs so that dosing in subsequent measurement of drug combinations is equipotent (e.g., the $I C_{00}$ should be dose \# 7 for all drugs). Doses may be spaced linearly or logarithmically, but the spacing must be consistent between drugs. The single-drug dose responses (blue and orange boxes) and the equipotent drug combination dose response (black box) are highlighted. Drug interactions can be estimated using only the measurements from these boxes rather than the entire checkerboard by approximating the shape of

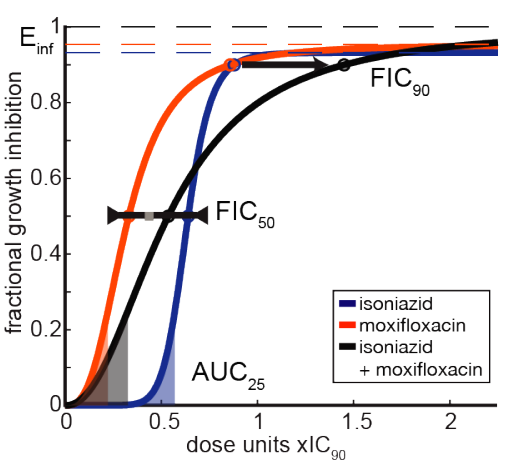
isoboles (contours of equal effect). In the diagram, the isobole for $\mathrm{IC}_{75}$ is traced by the circles. If drug $\mathrm{A}$ and $\mathrm{B}$ are additive, the isobole would be a straight diagonal, and we calculate the expected $\mathrm{IC}_{75}$ on the combination dose response (orange square) where the dotted line intersects with the diagonal (combination) dose response curve. In this illustration, the combination reaches an $\mathrm{IC}_{75}$ at higher dose levels (orange circle) than the expected IC , indicating an antagonistic interaction. The ratio of observed and expected doses (observed/expected) is the fractional inhibitory concentration (FIC):

$$
\mathrm{FIC}=\frac{\text { observed combination dose }}{\text { expected combination dose }}
$$

The DiaMOND methodology was used to obtain dose response data for every drug and drug combination measured over multiple time points. A Hill function was fit to these data and several potency and drug interactions metrics were derived from these dose response curves.

\section{DiaMOND dose response metrics:}

$\mathbf{E}_{\text {int }}$ (the maximum achievable effect): derived from the fitted Hill function (lower pane, dashed lines, colored by single drug or drug combination), $E_{\text {int }}$ describes the maximal achievable effect (upper asymptote, dashed lines) of a given drug or drug combination at a particular time point, where the maximum possible effect is 1.

$\mathrm{AUC}_{25}$ : the area under the curve (AUC) simultaneously captures variation in potency and effect of a drug or drug combination, i.e., sensitivity to drug. $\mathrm{AUC}_{25}$ captures sensitivity to drug at concentrations with low growth inhibition. To compare low does potency to other drugs or drug combinations with different concentration ranges, we normalize the area by dividing by the $\mathrm{IC}_{25}$. The resulting $\mathrm{AUC}_{25}$ values range from $\mathrm{O}$ (no effect) and $\mathbf{1}$ (potent).

FIC: drug interactions measure the effect of combining drugs on drug potency, i.e., the dose required to achieve a specific effect. The fractional inhibitory concentration $(F I C)$ is the ratio of the observed combination dose (black circle) to achieve $X$ effect over the expected combination dose (grey square), where FIC $<1$ indicates synergy, FIC $>1$ indicates antagonism, and $\mathrm{FIC}=1$ indicates additivity. In this example, the $\mathrm{FIC}_{50}$ is approximately additive whereas the $\mathrm{FIC}_{90}$ is antagonistic, which is indicated by the relative position of the combination dose response (black) near $\left(\mathrm{IC}_{50}\right)$ and to the right $\left(\mathrm{IC}_{90}\right)$ of the single dose response curves. We log transformation FICs to balance such that $\log _{2} \mathrm{FIC}<0$ is synergistic and $\log _{2} \mathrm{FIC}>0$ is antagonistic.

$\mathbf{G R}_{\mathrm{inf}}$ : Derived from the growth rate curve (not shown here, see (55) for details), GRinf describes the maximal achievable effect of a drug or drug combination on the normalized growth rate, ranging between 1 and -1 , where $\mathrm{GR}(\mathrm{c})$ is between 0 and 1 in the case of partial growth inhibition, $\mathrm{GR}(\mathrm{c})=0$ in the case of complete cytostasis, and $\mathrm{GR}<0$ indicates cell death. This unitless metric describes the effect of a drug on cells independent of doubling time, enabling comparison of drug effect on cells in different growth conditions. 
Table 1.

\begin{tabular}{|c|c|}
\hline & drug and drug combinations: \\
\hline B & bedaquiline \\
\hline $\mathrm{C}$ & clofazimine \\
\hline $\mathrm{E}$ & ethambutol \\
\hline $\mathrm{H}$ & isoniazid \\
\hline $\mathrm{L}$ & linezolid \\
\hline M & moxifloxacin \\
\hline $\mathrm{Pa}$ & pretomanid \\
\hline $\mathrm{Z}$ & pyrazinamide \\
\hline $\mathrm{R}$ & rifampicin \\
\hline $\mathrm{P}$ & rifapentine \\
\hline $\mathrm{D}$ & delamanid \\
\hline $\mathrm{Su}$ & sutezolid \\
\hline $\mathrm{Sq}$ & SQ109 \\
\hline $\mathrm{PaMZ}$ & bedaquiline + pretomanid + moxifloxacin \\
\hline BPaMZ & bedaquiline + pretomanid + moxifloxacin + pyrazinamide \\
\hline HRZE & $\begin{array}{c}\text { isoniazid + rifampicin + pyrazinamide }+ \text { ethambutol - four drug standard of } \\
\text { care }\end{array}$ \\
\hline HRZ & isoniazid + rifampicin + pyrazinamide - three drug standard of care \\
\hline $\mathrm{BPaL}$ & bedaquiline + pretomanid + linezolid \\
\hline MRZ & moxifloxacin + rifampicin + pyrazinamide \\
\hline $\mathrm{RZ}$ & rifampicin + pyrazinamide \\
\hline HRZM & $\begin{array}{c}\text { isoniazid + rifampicin }+ \text { pyrazinamide }+ \text { moxifloxacin }- \text { ReMOX trial } \\
\text { combination }\end{array}$ \\
\hline MRZE & $\begin{array}{c}\text { moxifloxacin }+ \text { rifampicin }+\begin{array}{c}\text { pyrazinamide }+ \text { ethambutol }- \text { ReMOX trial } \\
\text { combination }\end{array} \\
\end{array}$ \\
\hline PHZM & $\begin{array}{l}\text { rifapentine }+ \text { isoniazid }+ \text { pyrazinamide }+ \text { moxifloxacin }- \text { Study } 31 \text { trial } \\
\text { combination }\end{array}$ \\
\hline PHZE & $\begin{array}{c}\text { rifapentine }+ \text { isoniazid }+ \text { pyrazinamide }+ \text { ethambutol }- \text { Study } 31 \text { trial } \\
\text { combination }\end{array}$ \\
\hline $\mathrm{R}-\mathrm{CHOP}$ & $\begin{array}{l}\text { rituximab }+ \text { cyclophosphamide }+ \text { doxorubicin hydrochloride }+ \text { vincristine } \\
\text { sulfate }+ \text { prednisone }- \text { anti-cancer drug combination }\end{array}$ \\
\hline & treatment outcome classification: \\
\hline $\mathrm{C} 0$ & as good or worse than standard of care (HRZE or HRZ) \\
\hline $\mathrm{C} 1$ & better than standard of care \\
\hline
\end{tabular}




\begin{tabular}{|c|c|}
\hline & mouse models: \\
\hline RMM & relapsing mouse model ${ }^{1}$ \\
\hline $\mathrm{BMM}$ & bactericidal mouse model $^{2}$ \\
\hline \multirow[t]{2}{*}{$\mathrm{BHeB}$} & bactericidal outcome in $\mathrm{C} 3 \mathrm{HeB} / \mathrm{FeJ}$ mouse strain $^{3}$ \\
\hline & in vitro models: \\
\hline $\mathrm{a}$ & acidic \\
\hline $\mathrm{b}$ & butyrate \\
\hline $\mathrm{c}$ & cholesterol $(0.05 \mathrm{mM})$ \\
\hline $\mathrm{d}$ & dormancy \\
\hline $\mathrm{h}$ & cholesterol-high $(0.2 \mathrm{mM})$ \\
\hline $\mathrm{i}$ & intracellular \\
\hline $\mathrm{s}$ & standard \\
\hline \multirow[t]{2}{*}{$\mathrm{V}$} & valerate \\
\hline & data and model metrics: \\
\hline $\mathrm{C}$ & constant time point \\
\hline $\mathrm{T}$ & terminal time point \\
\hline $\mathrm{CT}$ & constant and terminal time point are the same \\
\hline $\mathrm{IC}_{\mathrm{n}}$ & inhibitory concentration at $\mathrm{n} \%$ growth inhibition \\
\hline $\mathrm{FIC}_{\mathrm{n}}$ & fractional inhibitory concentration at $\mathrm{n} \%$ growth inhibition \\
\hline $\mathrm{AUC}_{25}$ & normalized area under the curve to the $25 \%$ inhibition point \\
\hline$E_{\text {inf }}$ & effect at infinite drug concentration (maximum achievable effect) \\
\hline $\mathrm{GR}_{\text {inf }}$ & $\begin{array}{l}\text { normalized growth inhibition effect at infinite drug concentration (maximum } \\
\text { achievable effect) }\end{array}$ \\
\hline ROC & receiver operator characteristic \\
\hline AUC & area under the ROC curve \\
\hline PR & precision-recall \\
\hline F1 & harmonic mean of the precision and recall \\
\hline
\end{tabular}

Table 1: Abbreviations used in this study. Abbreviations along with brief descriptions are listed.

${ }^{1}$ The RMM outcome assesses lasting cure months after cessation of drug treatment in the most commonly used mouse strains (e.g., BALB/c, C56BL/6, Swiss). ${ }^{2}$ The BMM outcome assesses reduction of bacterial burden immediately following drug treatment in the most commonly used 
bioRxiv preprint doi: https://doi.org/10.1101/2021.02.03.429579; this version posted February 6, 2021. The copyright holder for this preprint (which was not certified by peer review) is the author/funder, who has granted bioRxiv a license to display the preprint in perpetuity. It is made available under aCC-BY-NC-ND 4.0 International license.

mouse strains. ${ }^{3}$ The BHeB assesses reduction of bacterial burden immediately following drug treatment but in the pathologically distinct $\mathrm{C} 3 \mathrm{HeB} / \mathrm{FeJ}$ mouse strain. 